\title{
Numerical simulations of highly porous dust aggregates in the low-velocity collision regime
}

\section{Implementation and calibration of a smooth particle hydrodynamics code ${ }^{\star}$}

\author{
R. J. Geretshauser ${ }^{1}$, R. Speith ${ }^{1}$, C. Güttler ${ }^{2}$, M. Krause ${ }^{2}$, and J. Blum² \\ ${ }^{1}$ Institut für Astronomie und Astrophysik, Abteilung Computational Physics, Eberhard Karls Universität Tübingen, \\ Auf der Morgenstelle 10, 72076 Tübingen, Germany \\ e-mail: ralf.j.geretshauser@uni-tuebingen.de \\ 2 Institut für Geophysik und extraterrestrische Physik, Technische Universität zu Braunschweig, Mendelssohnstr. 3, \\ 38106 Braunschweig, Germany
}

Received 3 November 2009 / Accepted 22 December 2009

\section{ABSTRACT}

\begin{abstract}
Context. A highly favoured mechanism of planetesimal formation is collisional growth. Single dust grains hit each other with relative velocities produced by gas flows in the protoplanetary disc. They stick together with van der Waals forces and form fluffy aggregates up to a centimetre size. The mechanism responsible for any additional growth is unclear since the outcome of aggregate collisions in the relevant velocity and size regime cannot be investigated in the laboratory under protoplanetary disc conditions. Realistic statistics for dust aggregate collisions beyond decimetre size are required to obtain a deeper understanding of planetary growth.

Aims. By combining experimental and numerical efforts, we wish to calibrate and validate a computer program capable of accurately simulating the macroscopic behaviour of highly porous dust aggregates. After testing its numerical limitations thoroughly, we check the program especially for a realistic reproduction of the compaction, bouncing, and fragmentation behaviour. This demonstrates the validity of our code, which will be utilised to simulate dust aggregate collisions and accurately determine the fragmentation statistics in future work.

Methods. We adopt the smooth particle hydrodynamics (SPH) numerical scheme with extensions to the simulation of solid bodies and a modified version of the Sirono porosity model. Experimentally measured macroscopic material properties of $\mathrm{SiO}_{2} \mathrm{dust}^{\mathrm{are}}$ implemented. By simulating three different setups, we calibrate and test for the compressive strength relation (compaction experiment) and the bulk modulus (bouncing and fragmentation experiments). Data from experiments and simulations will be compared directly. Results. SPH has already proven to be a suitable tool for simulating collisions at rather high velocities. In this work, we demonstrate that its area of application may be beyond low-velocity experiments and collisions. It can also be used to simulate the behaviour of highly porous objects in this velocity regime to very high accuracy. A correct reproduction of density structures in the compaction experiment, of the coefficient of restitution in the bouncing experiment, and of the fragment mass distribution in the fragmentation experiment illustrate the validity and consistency of our code for the simulation of the elastic and plastic properties of the simulated dust aggregates. The result of this calibration process is an SPH code that can be utilised to investigate the collisional outcome of porous dust in the low-velocity regime.
\end{abstract}

Key words. hydrodynamics - methods: laboratory - methods: numerical - planets and satellites: formation - protoplanetary disks

\section{Introduction}

In gaseous circumstellar discs, which are the potential birthplace of planetary systems, dust grains smaller than a micrometre grow to kilometre-sized planetesimals, which themselves proceed to terrestrial planets and cores of giant planets by gravity-driven runaway accretion. Depending on their size, the dust grains and aggregates move relative to each other in the disc. Brownian motion, radial drift, vertical settling, and turbulent mixing cause mutual collisions (Weidenschilling 1977; Weidenschilling \& Cuzzi 1993).

Since real protoplanetary dust particles are unavailable for experiments in the laboratory, much of the following work has been carried out with dust analogues such as $\mathrm{SiO}_{2}$ (Blum \& Wurm 2008). Theoretical models also refer to microscopic and macroscopic properties of these materials. Initially the dust

$\star$ Two movies are available in electronic form at http://www . aanda.org grains hit and stick on contact by means of van der Waals forces (Heim et al. 1999). In this process, which has been investigated experimentally (Blum et al. 2000; Blum \& Wurm 2000; Krause \& Blum 2004) and numerically (Dominik \& Tielens 1997; Paszun \& Dominik 2006, 2008, 2009; Wada et al. 2007, 2008, 2009), they form fluffy aggregates with a high degree of porosity.

Due to restructuring, the aggregates gain a higher mass to surface ratio and reach higher velocities. Blum \& Wurm (2000, 2008) and Wada et al. (2008) showed that collisions among them lead to fragmentation and mass loss. Depending on the model of the protoplanetary disc, which provides the kinetic collision parameters, this means that direct growth ends at aggregate sizes of a few centimetres. However, Wurm et al. (2005) and Teiser \& Wurm (2009) demonstrated in laboratory experiments for the centimetre regime with low-porosity dust that the projectile can stick partially to the target at velocities higher than $20 \mathrm{~m} \mathrm{~s}^{-1}$. Thus, collisional growth beyond centimetre size seems to be 
possible and the exact outcome of the fragment distribution is crucial for the understanding of the growth mechanism.

Numerical models that try to combine elaborate protoplanetary disc physics with the dust coagulation problem (Weidenschilling et al. 1997; Dullemond \& Dominik 2005; Brauer et al. 2008; Zsom \& Dullemond 2008; Ormel et al. 2007, 2009) have to make assumptions about the outcome of collisions between dusty objects of all sizes and relative velocities. Since data for these collisions are hardly available, in the most basic versions of these models perfect sticking, and in more elaborate ones power-law fragment distributions from experiments and observations (Mathis et al. 1977; Davis \& Ryan 1990; Blum \& Münch 1993) are assumed. The results of similar simulations depend highly on the assumed fragmentation kernel. However, the given experimental references have been measured only for small aggregate sizes. The influence of initial parameters such as the rotation of the objects or their porosity have not been taken into account for the size regimes beyond centimetre size, although they might play an important role (Sirono 2004; Ormel et al. 2007).

A new approach to modelling the growth of protoplanetary dust aggregates was developed by Güttler et al. (2010) and Zsom et al. (2010), who directly implemented the results of dust aggregation experiments into a Monte Carlo growth model. They found that the bouncing of protoplanetary dust aggregates plays a major role in their evolution as it is able to inhibit further growth and changes their aerodynamic properties. Although their model relies on the most comprehensive database available of dust aggregate properties and their collisional behaviour, they were unable to make direct predictions for any arbitrary set of collision parameters and were thus obliged to perform extrapolations over many orders of magnitude. Some of these extrapolations are based on physical models that need to be supported by additional experiments. Where there is no data available from experiments, the extrapolations have to be supported by sophisticated numerical models such that presented here.

Sticking, bouncing, and fragmentation are the important collision outcomes that need to be implemented into a coagulation code and affect the results of these models. Thus, it is not only important to correctly implement the exact thresholds between these regimes but also details concerning the outcome such as the fragment size distribution, the compaction in bouncing collisions, and maybe even the shape of the aggregates after they have merged in a sticking collision. Due to the lack of important input information, a systematic study of all relevant collision parameters is required and is addressed in this work.

Because of restrictions in size and realistic environment parameters, this task cannot be achieved in the laboratory alone. Much work has been completed on modelling the behaviour of dust aggregates on the basis of molecular interactions between the monomers (Paszun \& Dominik 2006, 2008, 2009; Wada et al. 2007, 2008, 2009). However, simulating dust aggregates with a model based on macroscopic material properties such as density, porosity, bulk and shear moduli, and compressive, tensile, and shear strengths remains an open field since these quantities are rarely available. The advantage of this approach over the molecular dynamics method, which is computationally limited to a few ten thousand monomers, is the accessibility of aggregate sizes beyond the centimetre regime.

Jutzi et al. (2008) implemented a porosity model into the smooth particle hydrodynamics (SPH) code by Benz \& Asphaug (1994). It was calibrated for pumice material using high-velocity impact experiments (Jutzi et al. 2009) and utilised to understand the formation of an asteroid family (Jutzi 2008). However, pumice is a material whose strength parameters decrease when it is compacted (crushed). Additionally, the underlying thermodynamically enhanced porosity model is designed to describe impacts of some $\mathrm{km} \mathrm{s}^{-1}$. Thus, it is perfectly suitable for simulating high-velocity collisions of porous rock-like material.

In contrast, collisions between pre-planetesimals occur at relative velocities of some tens of $\mathrm{m} \mathrm{s}^{-1}$ and compressive, shear, and tensile strengths increase with increasing density (Blum \& Schräpler 2004; Blum et al. 2006; Güttler et al. 2009).

Schäfer et al. (2007) used an SPH code based on the porosity model by Sirono (2004) to simulate collisions between porous ice in the $\mathrm{m} \mathrm{s}^{-1}$ regime. They found that a suitable choice of relations for the material parameters can produce sticking, bouncing, or fragmentation of the colliding objects. Therefore, they emphasised the importance of calibrating the material parameters of porous matter with laboratory measurements. Numerical molecular-dynamics simulations are almost capable of using a sufficient number of monomers to be close enough to the continuum limit and to provide the required material parameters (e.g., Paszun \& Dominik 2008; reproducing experimental results of Blum \& Schräpler 2004). They represent an important support to the difficult experimental determination of these quantities. In Güttler et al. (2009), we measured the compressive strength relation for spherical $\mathrm{SiO}_{2}$ dust aggregates for the static case in the laboratory and provided a prescription of how to apply this to the dynamic case using a compaction calibration experiment and 2D simulations. We pointed out relevant benchmark features and demonstrated how the code is in principal capable of simulating not only fragmentation but also bouncing, which has not yet been observed in molecular-dynamics codes.

In this paper, we present our SPH code with its technical details (Sect. 2), experimental reference (Sect. 3), and numerical properties (Sect. 4). On the basis of the compaction calibration simulation, we demonstrate that the results converge for increasing spatial resolution and choose a sufficient numerical resolution (Sect. 4.2). We investigate the differences between 2D and 3D numerical setup (Sect. 4.3) and thereby improve some drawbacks in Güttler et al. (2009). Artificial viscosity is presented as a stabilising tool for various problems in the simulations and its influence on the physical results is highlighted (Sect. 4.4).

Most prominently, we continue the calibration process started in Güttler et al. (2009) utilising two additional calibration experiments for bouncing (Sect. 5.2) and fragmentation (Sect. 5.3). In the end, we possess a collection of material parameters, which is consistent for all benchmark experiments. Finally, the SPH code has gained enough reliability to be used to enhance our information about the underlying physics of dust aggregate collisions beyond the centimetre regime. In future work, it will be applied to generate a catalogue of pre-planetesimal collisions and their outcome regarding all relevant parameters for planet formation. Jointly with experiments and coagulation models, this can be implemented into protoplanetary growth simulations (Güttler et al. 2010; Zsom et al. 2010) to enhance their reliability and predictive power.

\section{Physical model and numerical method}

\subsection{Smooth particle hydrodynamics}

The numerical Lagrangian particle method of smooth particle hydrodynamics (SPH) was originally introduced by Lucy (1977) and Gingold \& Monaghan (1977) to model compressible hydrodynamic flows in astrophysical applications. The method was later extended (Libersky \& Petschek 1990) and improved 
extensively (e.g., Libersky et al. 1993; Benz \& Asphaug 1994; Randles \& Libersky 1996; Libersky et al. 1997) to simulate elastic and plastic deformations of solid materials. A comprehensive description of SPH and its extensions can be found in Monaghan (2005).

In the SPH scheme, continuous solid objects are discretized into interacting mass packages of so-called "particles". These particles form a natural frame of reference for any deformation and fragmentation that the solid body may undergo. All spatial field quantities of the object are approximated onto the particle positions $\boldsymbol{x}_{i}$ by a discretized convolution with a kernel function $W$. The kernel $W$ depends on particle distance $\left|\boldsymbol{x}_{j}-\boldsymbol{x}_{i}\right|$ and has compact support, determined by the smoothing length $h$. We use the standard cubic spline kernel (Monaghan \& Lattanzio 1985) but normalised such that its maximum extension is equal to one smoothing length $h$.

We apply a constant smoothing length. This also allows us to model the fragmentation of solid objects in a simple way. Fragmentation occurs when some SPH particles within the body lose contact with their adjacent particles. Two fragments are completely separated as soon as their respective subsets of particles reach a distance of more than $2 h$ so that their kernels do not overlap any more.

Time evolution of the SPH particles is computed according to the Lagrangian form of the equations of continuum mechanics, by transferring spatial derivatives by means of partial integration onto the analytically given kernel $W$.

\subsection{Continuum mechanics}

A system of three partial differential equations forms the framework of continuum mechanics. As commonly known they follow from the constraints of conservation of mass, momentum, and energy. Accounting for the conservation of mass, the first is called the continuity equation

$\frac{\mathrm{d} \rho}{\mathrm{d} t}+\rho \frac{\partial v^{\alpha}}{\partial x^{\alpha}}=0$.

Following the usual notation, $\rho$ and $v^{\alpha}$ denote density and velocity, respectively. Greek indices run from 1 to $d$, the dimension of the problem. Einstein summing notation is used throughout the entire paper. In contrast to the usual SPH scheme, where the SPH density $\rho_{i}$ is calculated directly from the particle distribution, we solve the continuity equation according to

$\frac{\mathrm{d} \rho_{i}}{\mathrm{~d} t}=-\rho_{i} \sum_{j} \frac{m_{j}}{\rho_{j}}\left(v_{j}^{\alpha}-v_{i}^{\alpha}\right) \frac{\partial W^{i j}(h)}{\partial x_{i}^{\alpha}}$

(e.g., Randles \& Libersky 1996). Here the sum runs over all interaction partners $j$ of particle $i, m_{j}$ is the particle mass of particle $j$, and $W^{i j}$ denotes the kernel for the particular interaction. Although this approach is more expensive, as it requires the solution of an additional ordinary differential equation for each particle, it is more stable for high density contrasts and avoids artifacts caused by smoothing at boundaries and interfaces.

The conservation of momentum is ensured by the second equation

$\frac{\mathrm{d} v^{\alpha}}{\mathrm{d} t}=\frac{1}{\rho} \frac{\partial \sigma^{\alpha \beta}}{\partial x^{\beta}}$.

In SPH formulation, the momentum equation reads

$\frac{\mathrm{d} v_{i}^{\alpha}}{\mathrm{d} t}=\sum_{j} m_{j}\left(\frac{\sigma_{i}^{\alpha \beta}}{\rho_{i}^{2}}+\frac{\sigma_{j}^{\alpha \beta}}{\rho_{j}^{2}}\right) \frac{\partial W^{i j}(h)}{\partial x_{i}^{\beta}}$.
Because of the symmetry in the interaction terms, conservation of momentum is ensured by construction. In addition, we apply the standard SPH artificial viscosity (Monaghan \& Gingold 1983). This is essential in particular for stability at interfaces with highly varying densities. The influence of artificial viscosity on our simulation results is investigated thoroughly in Sect. 4.4.

The third equation, the energy equation, is not used in our model. Hence, we assume that kinetic energy is mainly converted into deformation energy and energy dissipated by viscous effects is converted into heat and radiated away. The stress tensor $\sigma$ can be devided into a part representing the pure hydrostatic pressure $p$ and a traceless part for the shear stresses, the so-called deviatoric stress tensor $S^{\alpha \beta}$. Hence,

$\sigma^{\alpha \beta}=-p \delta^{\alpha \beta}+S^{\alpha \beta}$

Any deformation of a solid body leads to a development of internal stresses in a specifically material-dependent manner. The relation between deformation and stresses is not taken into account within the regular equations of fluid dynamics, which are therefore insufficient for describing a perfectly elastic body and have to be extended. The missing relations are the constitutive equations, which depend on the strain tensor

$\epsilon^{\alpha \beta}=\frac{1}{2}\left(\frac{\partial x^{\prime \alpha}}{\partial x^{\beta}}+\frac{\partial x^{\prime \beta}}{\partial x^{\alpha}}\right)$

This represents the local deformation of the body. The primed coordinates denote the positions of the deformed body.

Following Hooke's law a proportional relation between deformation is assumed involving the material-dependent shear modulus $\mu=\mu(\rho)$, which depends itself on the density:

$S^{\alpha \beta} \propto 2 \mu\left(\epsilon^{\alpha \beta}-\frac{1}{d} \delta^{\alpha \beta} \epsilon^{\gamma \gamma}\right)$.

However, this is only the constitutive equation for the traceless shear part. For the hydrostatic part of the stress tensor, we adopt a modification of the Murnaghan equation of state, which is part of the Sirono (2004) porosity model:

$p(\rho)=K\left(\rho_{0}^{\prime}\right)\left(\rho / \rho_{0}^{\prime}-1\right)$,

where $\rho_{0}^{\prime}$ is the so called reference density, the density of the material at zero external stress, and $K(\rho)$ is the bulk modulus.

The density dependence of the bulk $K(\rho)$ and shear $\mu(\rho)$ moduli is modelled by a power law

$K(\rho)=2 \mu(\rho)=K_{0}\left(\rho / \rho_{i}\right)^{\gamma}$.

Although according to Sirono (2004) $\rho_{i}$ is the initial density of the material at the beginning of the simulation, we, in contrast, wish to ensure that our dust material possesses the same bulk modulus $K(\rho)$ even for simulations with different initial densities. In this work, the dust material has two different densities at the beginning of the bouncing (Sect. 5.2) and fragmentation (Sect. 5.3) calibration setup. According to Sirono (2004), the materials should feature two different $\rho_{i}$. As a consequence, $K(\rho)$, and in particular $K_{0}$, depends on the initial setup. Since we want to compare and validate $K_{0}$ by using two different setups, we have to fix a unique $\rho_{i}$ for all simulations. We choose $\rho_{i}$ such that $K\left(\rho_{i}\right)=K_{0}$ is the bulk modulus of the generic uncompressed dust material that is produced by the random ballistic deposition (RBD) method (Blum \& Schräpler 2004).

The time evolution of the pressure is directly given by the time evolution of the density (Eq. (1)) and since the pressure 
is a scalar quantity it is intrinsically invariant under rotation. However, to gain a frame-invariant formulation of the time evolution of the deviatoric stress tensor, i.e., the stress rate, correction terms have to be added. A very common formulation for SPH (see e.g., Benz \& Asphaug 1994; Schäfer et al. 2007) is the Jaumann rate form

$\frac{\mathrm{d} S^{\alpha \beta}}{\mathrm{d} t}=2 \mu\left(\dot{\epsilon}^{\alpha \beta}-\frac{1}{d} \delta^{\alpha \beta} \dot{\epsilon}^{\gamma \gamma}\right)+S^{\alpha \gamma} R^{\gamma \beta}+S^{\beta \gamma} R^{\gamma \alpha}$,

where $R^{\alpha \beta}$ is the rotation rate tensor, defined by

$R^{\alpha \beta}=\frac{1}{2}\left(\frac{\partial v^{\alpha}}{\partial x^{\beta}}-\frac{\partial v^{\beta}}{\partial x^{\alpha}}\right)$

and $\dot{\epsilon}^{\alpha \beta}$ denotes the strain rate tensor

$\dot{\epsilon}^{\alpha \beta}=\frac{1}{2}\left(\frac{\partial v^{\alpha}}{\partial x^{\beta}}+\frac{\partial v^{\beta}}{\partial x^{\alpha}}\right)$.

To determine rotation rate and strain rate tensor and thus the evolution of the stress tensor Eq. (10) in SPH representation, the SPH velocity derivatives $\partial v_{i}^{\alpha} / \partial x_{i}^{\beta}$ have to be calculated. The standard SPH formulation, however, does not conserve angular momentum because of the discretization error caused by particle disorder, which leads to rotational instability and in particular inhibits modelling rigid rotation of solid bodies. To avoid this error, we apply a correction tensor $C^{\gamma \beta}$ (Schäfer et al. 2007) according to

$\frac{\partial v_{i}^{\alpha}}{\partial x_{i}^{\beta}}=\sum_{j} \frac{m_{j}}{\rho_{j}}\left(v_{j}^{\alpha}-v_{i}^{\alpha}\right) \sum_{\gamma} \frac{\partial W^{i j}}{\partial x_{i}^{\gamma}} C^{\gamma \beta}$,

where the correction tensor $C^{\gamma \beta}$ is the inverse of

$\sum_{j} \frac{m_{j}}{\rho_{j}}\left(x_{i}^{\alpha}-x_{j}^{\alpha}\right) \frac{\partial W^{i j}}{\partial x_{i}^{\gamma}}$,

that is

$\sum_{j} \frac{m_{j}}{\rho_{j}}\left(x_{j}^{\alpha}-x_{i}^{\alpha}\right) \sum_{\gamma} \frac{\partial W^{i j}}{\partial x_{i}^{\gamma}} C^{\gamma \beta}=\delta^{\alpha \beta}$.

This approach leads by construction to first order consistency where the errors caused by particle disorder cancel out and the conservation of angular momentum is ensured. Only this allows rigid rotation to be simulated correctly.

Finally, the sound speed of the material is given by

$c_{\mathrm{S}}\left(\rho_{0}^{\prime}\right)=\sqrt{K\left(\rho_{0}^{\prime}\right) / \rho_{0}^{\prime}}$.

Together with Eq. (9), this relation shows that the sound speed is a strong function of density. This behaviour has been seen in molecular dynamics simulations by Paszun \& Dominik (2008), but there is no data available from laboratory measurements.

Up to this point the set of equations describes a perfectly elastic solid body. Additionally, the material simulated in this work are $\mathrm{SiO}_{2}$ dust aggregates, which have a high degree of porosity and, thus, plasticity. The modifications that account for these features are described in the next section.

\subsection{Porosity and plasticity}

Following the Sirono (2004) model, the porosity $\Phi$ is modelled by the density of the porous material $\rho$ and the constant matrix density $\rho_{\mathrm{s}}$

$\Phi=1-\frac{\rho}{\rho_{\mathrm{s}}}=1-\phi$.

In the following, we use the filling factor $\phi=\rho / \rho_{\mathrm{s}}$.

We model plasticity by reducing inner stresses given by $\sigma^{\alpha \beta}$. For this reason, we need constitutive relations describing the behaviour of the material during plastic deformation. These relations are specific for each material and have to be determined empirically. For highly porous materials it is particularly difficult to acquire them. Therefore, it is advantageous that the model is based on measurements by Blum \& Schräpler (2004), Blum et al. (2006), and Güttler et al. (2009).

The main idea of the adopted plasticity model is to reduce inner stress once the material exceeds a certain plasticity criterion. In the elastic case, described by Eqs. (7) and (8), inner stresses grow linearly with deformation. Hence, the material returns to its original shape at vanishingly external forces. Reducing inner stresses, i.e., deviating from the elastic deformation path, reduces the internal ability of the material to restore its original shape. Therefore, by means of stress reduction, deformation becomes permanent, i.e., plastic. Following and expanding the approach by Sirono (2004), we treat the plasticity of the pure hydrostatic pressure $p$ and the deviatoric stress tensor $S^{\alpha \beta}$ separately.

For the deviatoric stress tensor, we follow the approach by Benz \& Asphaug (1995) and Schäfer et al. (2007) by assuming that our material is isotropic, which makes the von Mises yield criterion applicable. This criterion is characterised by the shear strength $Y$, which in our model is a composite of the compressive and tensile strengths $Y(\phi)=\sqrt{\Sigma(\phi)|T(\phi)|}$. The suitability of this choice was already demonstrated in Güttler et al. (2009). Since $Y(\phi)$ is a scalar, we have to derive a scalar quantity from $S^{\alpha \beta}$ for reasons of comparability. We do this by calculating its second irreducible invariant $J_{2}=S^{\alpha \beta} S^{\alpha \beta}$. Finally, the reduction of the deviatoric stress is implemented in the following way $S^{\alpha \beta} \rightarrow f S^{\alpha \beta}$,

where $f=\min \left[Y^{2}(\phi) / 3 J_{2}, 1\right]$. The hydrostatic pressure is limited by the tensile strength $T(\phi)$ for $p<0$ and by the compressive strength $\Sigma(\phi)$ for $p>0$ :

$p(\phi)= \begin{cases}\Sigma(\phi) & \phi>\phi_{\mathrm{c}}^{+} \\ T(\phi) & \phi<\phi_{\mathrm{c}}^{-}\end{cases}$

For $\phi_{\mathrm{c}}^{-} \leq \phi \leq \phi_{\mathrm{c}}^{+}$, the material is in the elastic regime and Eq. (8) is applied. The symbols $\phi_{\mathrm{c}}^{-}$and $\phi_{\mathrm{c}}^{+}$denote the filling factors where the elastic path intersects the tensile strength and compressive strength, respectively (see Fig. 1).

The pressure reduction process is implemented such that, at each time step, $p$ is computed using Eq. (8). If, for a given, $\phi$ $p(\phi)>\Sigma(\phi)$ and $\phi>\phi_{\mathrm{c}}^{+}$, then the pressure $p(\phi)$ is reduced to $\Sigma(\phi)$. The deformation becomes irreversible once the new reference density $\rho_{0}^{\prime}$ is computed using Eq. (8) and the elastic path is shifted towards higher densities. Hereby the limiting filling factors $\phi_{\mathrm{c}}^{-}$and $\phi_{\mathrm{c}}^{+}$are also set anew. In principal, there are two possible implementations of this: (1) plasticity becomes effective immediately and $\rho_{0}^{\prime}$ is computed whenever $p>\Sigma$; and (2) plasticity becomes effective after pressure decrease, which is equivalent to $\phi<\phi_{\mathrm{c}}^{+}$. We tested both implementations. For our understanding, possibility (1) is closer to the underlying physical 


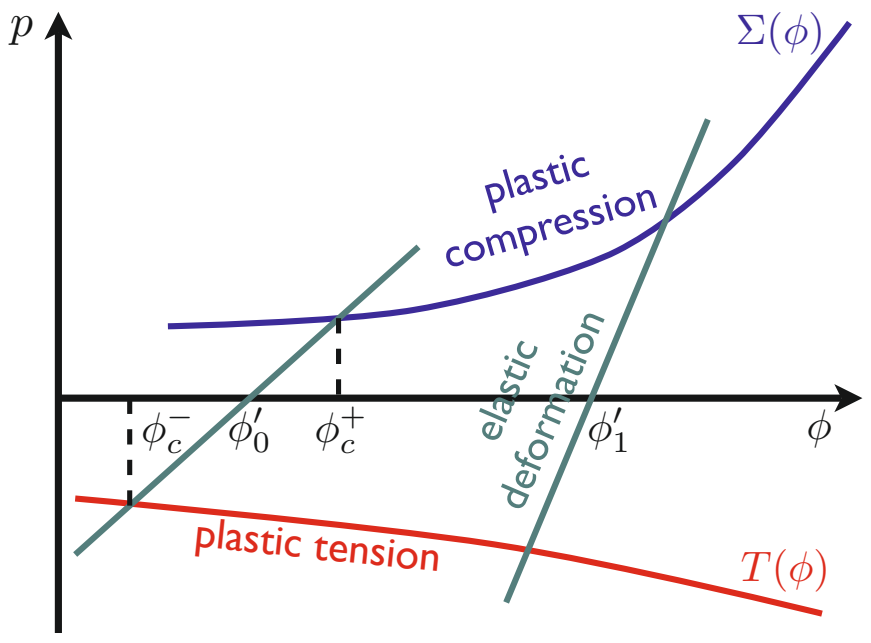

Fig. 1. In the modified Sirono porosity model the regime of elastic deformation is limited by the compressive strength $\Sigma(\phi)$, which represents the transition threshold to plastic compression for $p>\Sigma(\phi)$, and the tensile strength $T(\phi)$, which represents the transition threshold to plastic tension or rupture for $p<T(\phi)$. Returning from one of the plastic regimes to vanishing external pressure via an elastic path leads to a $\phi_{1}^{\prime}$ that differs from the initial $\phi_{0}^{\prime}$. Hence, the material has been deformed irreversibly.

process. In addition, it has proven to be more stable. According to the benchmark parameters, the results were equivalent.

For the tensile regime, i.e., for $\phi<\phi_{\mathrm{c}}^{-}$, we do not adopt the damage and damage-restoration model presented in Sirono (2004). This damage model for brittle material such as rocks or pumice was developed for SPH by Benz \& Asphaug (1994, 1995) and used by Jutzi (2008) and Jutzi et al. (2008, 2009). It is assumed that a material contains flaws, which are activated and develop under tensile loading (Grady \& Kipp 1980). Schäfer et al. (2007) found that the model is not applicable to their simulations of porous ice because it includes compressive damage effects. Brittle material such as pumice and rocks tend to disintegrate when compressed, i.e., they are crushed. In contrast in our material of highly porous $\mathrm{SiO}_{2}$ dust, both the tensile and compressive strengths increase with compression. This is because the monomers are able to form new bonds when they come into contact. Therefore, we adopt the same approach as in the compressive regime and reduce the pressure $p(\phi)$ to $T(\phi)$ once the tensile strength is exceeded. Finally, the material can rupture because of the plastic flow. However, material that is plastically stretched can be compressed again up to its full strength. By choosing this approach, a "damage restoration model" is implemented in a very natural way.

Finally, a remark has to be made about energy. Apart from energy dissipation by numerical and artificial viscosity, we assume intrinsic energy conservation. We suppose that heat production in the investigated physical processes is negligible. Therefore, our model is limited to a velocity regime below the sound speed $c_{\mathrm{s}}$ of the dust material $(\approx 30 \mathrm{~m} / \mathrm{s}$, see Blum \& Wurm 2008; Paszun \& Dominik 2008). By choosing the approach to modelling plasticity that involves stress reduction, we assume that most of the energy is dissipated by plastic deformation, since the reduction in internal stresses accounts for the reduction in internal energy.

Since we do not solve the energy equation thermodynamically enhanced features such as any phase transition such as melting and freezing cannot be simulated. This scheme also does not feature a damage model. When considering fragmentation (Sect. 5.3) in particular, any flaws in the material cannot yet be taken into account, although they might influence the resulting fragment distribution.

\section{Experimental reference}

\subsection{Material parameters}

The material used in the calibration experiments are highlyporous dust aggregates as described by Blum \& Schräpler (2004), consisting of spherical $\mathrm{SiO}_{2}$ spheres with a diameter of $1.5 \mu \mathrm{m}$. For these clearly defined dust aggregates, it is possible to reproducibly measure macroscopic material parameters such as tensile strength, compressive strength, and, potentially, also the shear strength needed for the SPH porosity model (see Sect. 2.3). The tensile strength of this material was measured for highly porous and compacted aggregates $(\phi=0.15 \ldots 0.66)$ by Blum $\&$ Schräpler (2004). These measurements support a linear dependence between the tensile strength and the number of contacts per monomer (increasing with increasing $\phi$ ), which yields the tensile strength as

$T(\phi)=-\left(10^{2.8+1.48 \phi}\right) \mathrm{Pa}$.

The compressive strength was measured in the experimental counterpart of this paper (Güttler et al. 2009) with an experimental setup to determine the static omni-directional compression (ODC), whereby the sample is enclosed from all sides and the pressure is constant within the sample. We found that the compressive strength curve can be well described by the analytic function

$\Sigma(\phi)=p_{\mathrm{m}} \cdot\left(\frac{\phi_{2}-\phi_{1}}{\phi_{2}-\phi}-1\right)^{\Delta \cdot \ln 10}$,

where the free parameters were measured to be $\phi_{1}=0.12$, $\phi_{2}=0.58, \Delta=0.58$, and $p_{\mathrm{m}}=13 \mathrm{kPa}$. However, these parameters were measured with a static setup and we expect a different strength for a dynamic collision. The parameters $\phi_{1}$ and $\phi_{2}$ determine the range of the volume-filling factor, where $\phi_{1}$ is defined by the uncompressed material and $\phi_{2}$ corresponds to the densest packing. These parameters are expected to be the same as for the dynamic compression, while $p_{\mathrm{m}}$ and $\Delta$ are treated as free parameters, which we calibrate in the forthcoming sections. The last important material parameter to describe the dust aggregates is the elasticity. We can determine the Young's modulus from measurement and simulation of the sound speed (Blum \& Wurm 2008; Paszun \& Dominik 2008), which is $c_{\mathrm{s}}=30 \mathrm{~m} \mathrm{~s}^{-1}$. From this approach, the bulk modulus for the uncompressed material is $K_{0}=\rho_{\mathrm{i}} c_{\mathrm{s}}^{2}=300 \mathrm{kPa}$ with $\rho_{\mathrm{i}}=300 \mathrm{~kg} \mathrm{~m}^{-3}$. However, other plausible calculations (Weidling et al. 2009) indicate an elasticity of close to $1 \mathrm{kPa}$. We therefore also vary this parameter.

\subsection{Calibration experiment}

As a setup for an easy and well-defined calibration experiment (see Güttler et al. 2009), we chose a glass bead with a diameter of 1 to $3 \mathrm{~mm}$, which impacts into the dust aggregate material with a velocity of between 0.1 and $1 \mathrm{~m} \mathrm{~s}^{-1}$ under vacuum conditions (pressure 0.1 mbar). We were able to measure the deceleration curve, stopping time, and intrusion depth of the glass bead (for various velocities and projectile diameters) and the compaction of the dust under the glass bead (for a $1.1 \mathrm{~mm}$ projectile 
with a velocity of $0.65 \mathrm{~m} \mathrm{~s}^{-1}$ ). These results help us to calibrate and test the SPH code.

For the measurement of the deceleration curve, we used an elongated epoxy projectile instead of the glass bead. The bottom shape and the mass resembled the glass bead, while the lower density and the therefore longer extension made it possible to observe the projectile during the intrusion. The projectile was observed by a high-speed camera (12000 frames per second) and the position of the upper edge was followed with an accuracy of $\approx 3 \mu \mathrm{m}$. We found that - independently of velocity and projectile diameter - the intrusion curve can be described by a sine curve

$h(t)=-D \cdot \sin \left(\frac{\pi}{2} \frac{t}{T_{\mathrm{s}}}\right) ; \quad t \leq T_{\mathrm{s}}$,

where, $D$ and $T_{\mathrm{s}}$ denote the intrusion depth and the stopping time of the projectile, respectively. The stopping time is defined as the time between the first contact and the deepest intrusion at zero velocity. In Sect. 5.1.2, we use the normalised form of the intrusion curve with $D=T_{\mathrm{s}}=1$.

For the intrusion depth, we found good agreement with a linear behaviour of

$D=\left(8.3 \times 10^{-4} \frac{\mathrm{m}^{2} \mathrm{~s}}{\mathrm{~kg}}\right) \cdot \frac{m v}{A}$,

where $v$ is the impact velocity of the projectile and $m$ and $A=\pi r^{2}$ are the mass and cross-sectional area of the projectile, respectively. We found the stopping time $T_{\mathrm{s}}$ to be independent of the velocity and to depend only on the projectile size $(3.0 \pm 0.1 \mathrm{~ms}$ for $1 \mathrm{~mm}$ projectiles and $6.2 \pm 0.1 \mathrm{~ms}$ for $3 \mathrm{~mm}$ projectiles).

The compaction of dust underneath the impacted glass bead was measured by X-ray micro-tomography. The glass bead diameter and velocity in these experiments corresponded to the compaction calibration setup described in Table 1. The dust sample with an embedded glass bead was positioned onto a rotatable sample carrier between an X-ray source and the detector. During the rotation through $360^{\circ}$, 400 transmission images were taken, from which we computed a $3 \mathrm{D}$ density reconstruction with a spatial resolution of $21 \mu \mathrm{m}$. The corresponding results for the density reconstruction can be found in Güttler et al. (2009), where we found that roughly one sphere volume beneath the glass bead is compressed to a volume-filling factor of $\phi \approx 0.23$, while the surrounding volume is nearly unaffected with an original volume-filling factor of $\phi \approx 0.15$. In this work, we focus on the vertical density profile through the centre of the sphere and the compressed material (see Sect. 4).

\subsection{Additionaly benchmark experiments}

We present two additional experiments used to validate the SPH code in Sects. 5.2 and 5.3. Heißelmann et al. (2007) performed low-velocity collisions $\left(v=0.4 \mathrm{~m} \mathrm{~s}^{-1}\right)$ between cubicshaped, approximately $5 \mathrm{~mm}$-sized aggregates of the material as described in Sect. 3.1 and found bouncing whereby about $95 \%$ of the energy was dissipated in a central collision. Detailed investigation of the compaction in these collisions (Weidling et al. 2009 ) revealed significant compaction of the aggregates (from $\phi=0.15$ to $\phi=0.37$ ) after approximately 1000 collisions. The energy needed for this compaction is consistent with the energy dissipation measured by Heißelmann et al. (2007).

An additional experiment deals with the disruptive fragmentation of dust aggregates (for details, see Güttler et al. 2010, 2009). In this case, a dust aggregate with a diameter of $0.57 \mathrm{~mm}$ consisting of $1.5 \mu \mathrm{m}$ spherical $\mathrm{SiO}_{2}$ dust with a volume-filling
Table 1. Numerical parameters for the compaction calibration setup.

\begin{tabular}{lccl}
\hline \hline Physical quantity & Symbol & Value & Unit \\
\hline Glass bead & & & \\
Bulk density $^{(*)}$ & $\rho_{0}$ & 2540 & $\mathrm{~kg} \mathrm{~m}^{-3}$ \\
Bulk modulus $^{(*)}$ & $K_{0}$ & $5 \times 10^{9}$ & $\mathrm{~Pa}$ \\
Murnaghan exponent $^{(*)}$ & $n$ & 4 & - \\
Radius & $r$ & $0.55 \times 10^{-3}$ & $\mathrm{~m}$ \\
Impact velocity & $v_{0}$ & 0.65 & $\mathrm{~m} \mathrm{~s}^{-1}$ \\
\hline Dust sample & & & \\
Initial density & $\rho_{i}$ & 300 & $\mathrm{~kg} \mathrm{~m}^{-3}$ \\
Bulk density & $\rho_{\mathrm{s}}$ & 2000 & $\mathrm{~kg} \mathrm{~m}^{-3}$ \\
Reference density & $\rho_{0}^{\prime}$ & 300 & $\mathrm{~kg} \mathrm{~m}^{-3}$ \\
Initial filling factor & $\phi_{i}$ & 0.15 & - \\
Bulk modulus & $K_{0}$ & $3 \times 10^{5}$ & $\mathrm{~Pa}^{\prime}$ \\
ODC mean pressure & $p_{\mathrm{m}}$ & 1300 & $\mathrm{~Pa}$ \\
ODC max. filling factor & $\phi_{2}$ & 0.58 & - \\
ODC min. filling factor & $\phi_{1}$ & 0.12 & - \\
ODC slope & $\Delta$ & 0.58 & - \\
\hline
\end{tabular}

Notes. ODC stands for omni-directional compression relation (Eq. (21)). Quantities marked by $(*)$ represent the parameters for sandstone in Melosh (1989) which we adopt for glass here.

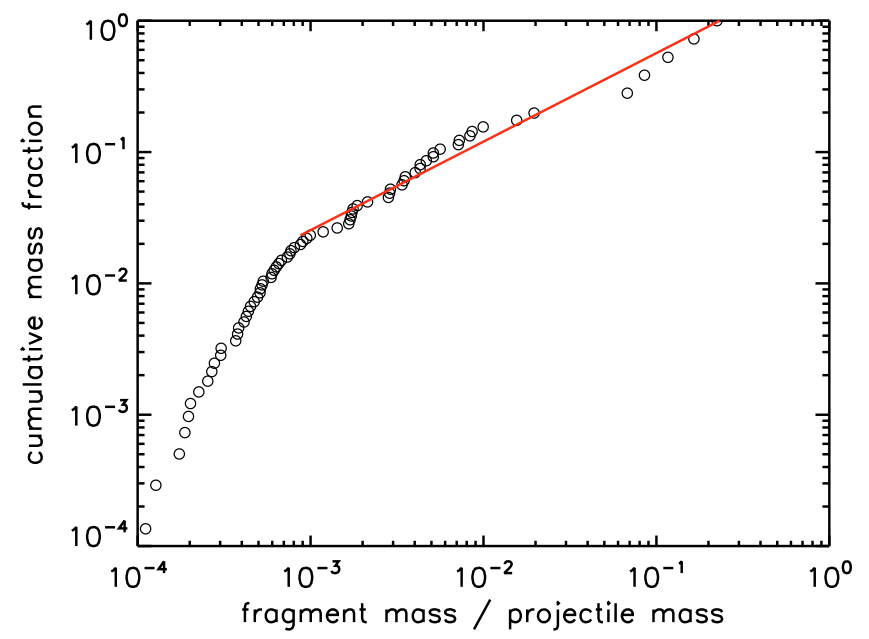

Fig. 2. Cumulative mass distribution of the fragments after a disruptive collision, which can be described by a power law. The divergence at low masses is caused by the depletion of small aggregates because of the camera resolution.

factor of $\phi=0.35$ collides with a solid glass target at a velocity of $8.4 \mathrm{~m} \mathrm{~s}^{-1}$ (also see Fig. 20 in Güttler et al. 2009). The projectile fragments and the projected sizes of these fragments are measured with a high-speed camera with a resolution of $16 \mu \mathrm{m}$ per pixel. As the mass measurement is restricted to the $2 \mathrm{D}$ images, the projected area of each fragment is averaged over a sequence of images, where it is clearly separated from other fragments. From this projected area, the fragment masses are calculated with the assumptions of a spherical shape and an unchanged volume filling factor. Figure 2 shows the mass distribution in a cumulative plot. For higher masses, which are not depleted by the finite camera resolution, we find good agreement with a power-law distribution

$n(m) \mathrm{d} m=\left(\frac{m}{\mu}\right)^{\alpha} \mathrm{d} m$,

where $m$ is the normalised mass (fragment mass divided by projectile mass) and $\mu=0.22$ is a measure of the strength of 
fragmentation, being defined as the mass of the largest fragment divided by the mass of the projectile. The exponent $\alpha$ has a value of 0.67. A similar distribution was already described by Blum \& Münch (1993) for aggregate-aggregate fragmentation of $\mathrm{ZrSiO}_{4}$ aggregates of comparable porosity.

\section{Numerical issues}

Before we perform the calibration process, some numerical issues have to be resolved. For instance, it is unfeasible to simulate the dust sample, into which the glass bead drops in the compaction calibration experiment presented in Sect. 3, as a whole. It is also infeasible to carry out all necessary computations in 3D. Therefore, we simulate only part of the dust sample and attempt to determine the size at which spurious boundary effects emerge. Most of the calibration process was conducted in $2 \mathrm{D}$, but the differences between $2 \mathrm{D}$ and $3 \mathrm{D}$ results are discussed and quantified.

In this context, we use the results of $2 \mathrm{D}$ simulations in cartesian coordinates, although the symmetry of the problem would be more accurately described by cylindrical coordinates. However, the SPH scheme in cylindrical or polar coordinates battles with the problem of a singularity at the origin of the kernel function. There have been only a few attempts to resolve this issue (e.g., Omang et al. 2006), which, however remain under development and require high implementation efforts. In our case, since 2D simulations provide only an indication of the calibration required and 3D simulations are aimed at, we stick to cartesian coordinates.

The glass bead is simulated with the Murnaghan equation of state

$p(\rho)=\left(\frac{K_{0}}{n}\right)\left[\left(\frac{\rho}{\rho_{0}}\right)^{n}-1\right]$,

following the usual laws of continuum mechanics as presented in Sect. 2.2. The compaction calibration setup is initialised with the numerical parameters shown in Table 1, unless stated otherwise in the text. Our tests showed that the maximum intrusion depth and the density profile are the calibration parameters most sensitive to changes in the numerical setup. Density profiles (e.g., Figs. 4 and 5) display the filling factor $\phi$ along a line through the centre of the sphere and perpendicular to the bottom of the dust sample (see Fig. 3). The origin of the diagrams represents the surface of the unprocessed dust sample. The glass sphere has been removed in these figures.

\subsection{Computational domain and boundary conditions}

In $2 \mathrm{D}$ simulations, we tested the effect of changing the size and shape of the dust sample. Initially the particles were set on a triangular lattice with a lattice constant of $25 \mu \mathrm{m}$. To be geometrically consistent with the cylindric experimental setup, we firstly utilised a box (width $8 \mathrm{~mm}$ ) and varied its depth: $1.375 \mathrm{~mm}$, $2.2 \mathrm{~mm}, 3.3 \mathrm{~mm}$, and $5.5 \mathrm{~mm}$. This is equivalent to the dimensions $2.5 \times, 4 \times, 6 \times$, and $10 \times r_{\text {sphere }}$ Comparing the density profiles (Fig. 4, top), two features are remarkable: (1) the maximum filling factor at the top of the dust sample $(\phi \approx 0.27$ at $D \approx-0.6 \mathrm{~mm}$ ) and the intrusion depth $D$ is nearly the same for all dust sample sizes. (2) For $d_{\text {sample }}<3.3 \mathrm{~mm}$, we find spurious density peaks at the lower boundaries $(D \approx-1.4 \mathrm{~mm}$ and $D \approx-2.2 \mathrm{~mm}$ ).

To reduce the computation time, we simulated the dust sample as a semicircle with the same radius variation as above. The

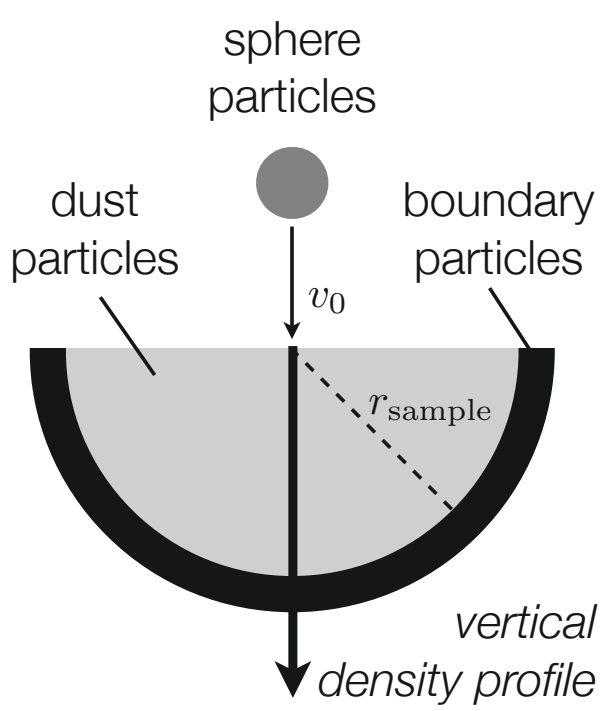

Fig. 3. Compaction calibration setup in 2D or, respectively, crosssection of 3D compaction calibration setup. A glass sphere impacts into a dust sample (radius $r_{\text {sample }}$ ) with initial velocity $v_{0}$. The dust sample is surrounded by boundary particles at its bottom. Their acceleration is set to zero at every time step. The vertical density profile at maximum intrusion serves as calibration feature.

resulting density profiles are shown in Fig. 4 (bottom). In contrast to the corresponding simulations with the box-shaped samples, we find for $r_{\text {sample }} \leq 1.375 \mathrm{~mm}$ an increased maximum filling factor and a slightly reduced intrusion depth. Because of the greater amount of volume lateral to the intrusion channel, material can be pushed aside more easily than inside the narrow boundaries of the semicircle. Therefore, a higher fraction of the material is compressed to higher filling factors. For $r_{\text {sample }}>$ $3.3 \mathrm{~mm}$, the spurious boundary effects become negligible within the compaction calibration setup and the density structure shows no significant difference for box-shaped and semicircle-shaped dust samples.

Hence, all computations of Sect. 4 are conducted on the basis of a semicircle in $2 \mathrm{D}$ or a hemisphere in $3 \mathrm{D}$ with a radius of $r_{\text {sphere }}=3.3 \mathrm{~mm}$.

In all cases, the dust sample is bordered by a few layers of boundary particles. The acceleration of these particles is set to zero at each integration step, simulating reflecting boundary conditions. Apart from that, i.e., in terms of the equation of state, they are treated like dust particles. We also tested damping boundary conditions by simulating two layers of boundaries. The outer layer was treated as described above, the inner (sufficiently large) layer was simulated with a high artificial $\alpha$-viscosity. Since there was no significant difference in the outcome, we fix all boundaries in the aforementioned way and consider them to be reflecting.

\subsection{Resolution and Convergence}

Jutzi (2008) found in his studies of a basalt sphere impacting a porous target that the outcome of his simulations strongly depended on their resolutions. With a calibration setup similar to that used in this paper, Geretshauser (2006) confirms that in simulations of the type presented in this work, a strong resolution dependence is present. He found that the intrusion depth of the glass bead can be doubled by doubling the resolution. Since the calibration experiments presented in Güttler et al. (2009) are extremely sensitive even to minor changes in the setup, 

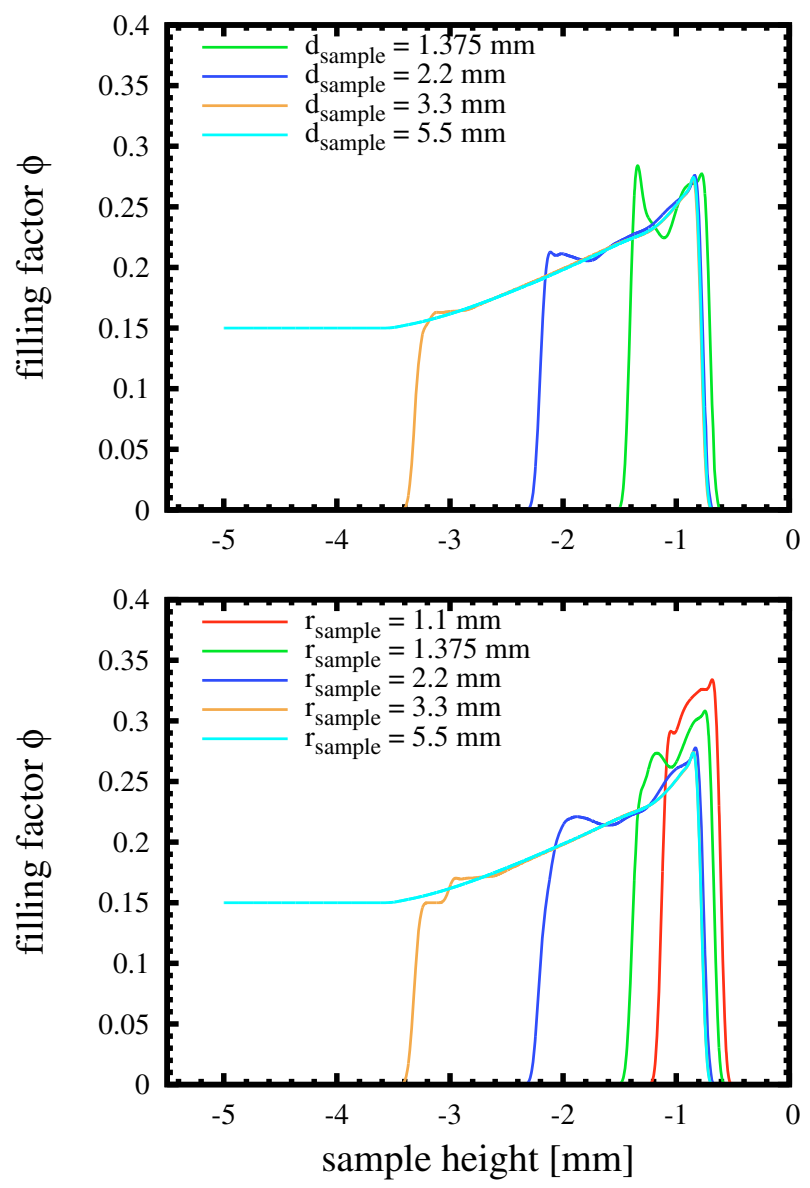

Fig. 4. Vertical density profile at maximum intrusion for the compaction calibration setup and different shapes of the 2D dust sample (box and semicircle). Depth $d_{\text {sample }}$ of an $8 \mathrm{~mm}$ wide box (top) and radius $r_{\text {sample }}$ of the semicircle (bottom) were varied. In both cases spurious boundary effects appear for $d_{\text {sample }}<3.3 \mathrm{~mm}$ and $r_{\text {sample }}<3.3 \mathrm{~mm}$, respectively.

the convergence properties of porosity model and the underlying SPH method are investigated carefully in this paragraph. Additionally, we study the differences in the outcome of 2D and 3D setup.

For the 2D convergence study, particles were initially placed on a triangular lattice again. The lattice constants $l_{\mathrm{c}}$ were 100 , 50,25 , and $12.5 \mu \mathrm{m}$ for the compaction calibration setup. The smoothing length $h$ was kept constant relative to $l_{\mathrm{c}}$ at a ratio of $5.6 \times l_{\mathrm{c}}$. The maximum number of interaction partners was $I_{\max } \approx 180$, the average $I_{\mathrm{av}} \approx 100$, and the minimum $I_{\min } \approx 30$.

In the $3 \mathrm{D}$ convergence study, we used a cubic lattice with edge lengths $l_{\mathrm{c}}=100,50$, and $25 \mu \mathrm{m}$. The latter was simulated with 3.7 million SPH particles, which represent the limit of our computational resources. We fixed $h=3.75 \times l_{\text {c }}$, which yielded $I_{\max } \approx 370, I_{\mathrm{av}} \approx 240$, and $I_{\min } \approx 70$. The results are presented in Fig. 5. In contrast to the plots in Fig. 4, the glass sphere is not removed here. Coming from the right side of the plot, the filling factor rapidly decreases from a high value beyond the edge of the plot describing the sphere. The filling factor reaches its minimum at an artificial gap between sphere and surface of the dust sample. The width of this gap is about one smoothing length $h$. The existence of the gap has two reasons: (1) sphere material and dust material have to be separated by artificial viscosity for stability reasons. This is discussed below. (2) The volume of the sphere represents an area of extremely high density and pressure with respect to the dust
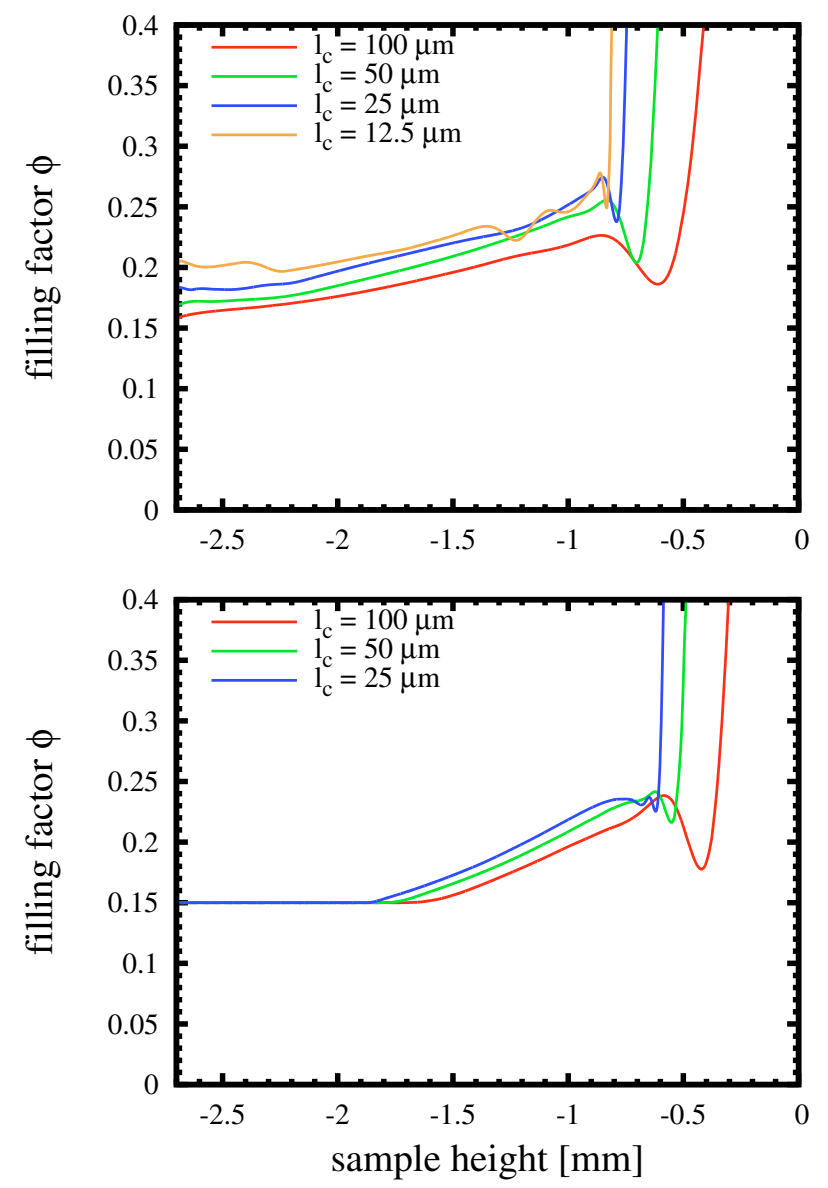

Fig. 5. Convergence study of the vertical density profile for 2D (top) and $3 \mathrm{D}$ (bottom) compaction calibration setups. The increase in filling factor towards the surface of the dust sample accounts for the glass bead, which is not removed in this plot. Simulations were performed for different spatial resolutions. All curves show a characteristic fillingfactor minimum between the sphere and dust sample and a characteristic filling-factor maximum indicating the dust sample surface.

sample. This area is smoothed out by the SPH method, the width of the smoothing being given by the smoothing length. Although clear convergence behaviour is evident in Fig. 5 for both the 2D and the $3 \mathrm{D}$ case, a more unique convergence criterion has to be found. For this purpose, we choose the maximum intrusion depth, which proved to be very sensitive to resolution changes. The shape of the filling-factor profile provides two ways to determine the intrusion depth: (1) the filling factor minimum, which is inbetween sphere and dust sample; and (2) the filling factor maximum (peak) of the dust material left in the gap between sphere and dust sample.

Figure 6 shows the results for both cases in 2D (top) and $3 \mathrm{D}$ (bottom). The error bars around the minimum values represent the smoothing length and provide an indication of the maximum error. The position of the density peak remains almost constant, converging to $\approx-0.9 \mathrm{~mm}(2 \mathrm{D})$ and $\approx-0.65 \mathrm{~mm}(3 \mathrm{D})$, respectively, at higher resolutions. The position of the density minimum at low resolutions differs significantly from the position of the density peak, but converges quickly to the same intrusion depth at higher resolutions. However, the differences between the extrema remain well within one smoothing length. This is because the sphere and dust sample are separated by about one smoothing length. Comparing 2D and 3D convergence, the $3 \mathrm{D}$ case seems to converge more quickly. 

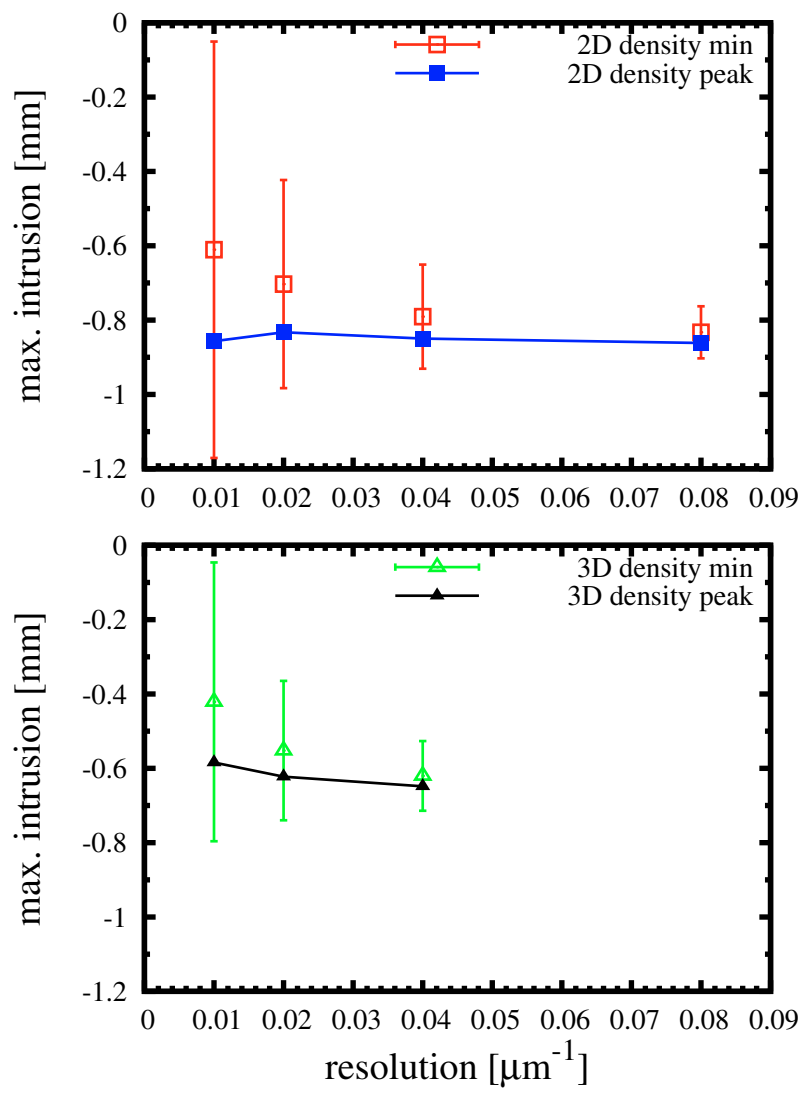

Fig. 6. Convergence study of the maximum intrusion depth for the 2D (top) and 3D (bottom) compaction calibration setups. Filled symbols represent the position of the filling factor peak of the dust material, whereas empty symbols denote the position of the minimum filling factor at the gap between glass bead and dust material. The values are derived from the density profiles in Fig. 5. The smoothing length is indicated by the error bars. While the peak position remains almost constant at $\approx-0.9 \mathrm{~mm}(2 \mathrm{D})$ and $\approx-0.65 \mathrm{~mm}$ (3D) with increasing spatial resolution, the position of the filling-factor minimum quickly converges to the same value. This is because of the artificial separation between dust and glass materials, which is of the order of a smoothing length.

Because of the findings of this study, we choose a spatial resolution of $l_{\mathrm{c}}=25 \mu \mathrm{m}$ for additional simulations in 2D. In the $3 \mathrm{D}$ case, $l_{\mathrm{c}}=50 \mu \mathrm{m}$ is sufficient, but $l_{\mathrm{c}} \leq 50 \mu \mathrm{m}$ is desirable if feasible. After defining suitable values for the spatial resolution, we now turn to the numerical resolution, which for the SPH scheme is given by the number of interaction partners of each single particle. For the investigation of this feature, we performed a study utilising the $2 \mathrm{D}$ compaction calibration setup with a spatial resolution of $l_{\mathrm{c}}=25 \mu \mathrm{m}$ and varied the ratio of smoothing length to lattice constant $h / l_{\mathrm{c}}$ from 2 to 7 in steps of one, where $h / l_{\mathrm{c}}$ determines the initial number of interaction partners that is smoothed over. The resulting maximum, average, and minimum interactions $I_{\max }, I_{\mathrm{av}}$, and $I_{\min }$ and the corresponding smoothing lengths $h$ can be found in Table 2 . Additionally, we measured the time $T_{\text {comp }}$ the computations took, simulated on 4 cores of a cluster with Intel Xenon QuadCore processors $(2.66 \mathrm{GHz})$ for a simulated time of $5 \mathrm{~ms}$ and the number of integration steps $N_{\text {step }}$ of our adaptive Runge-Kutta Cash-Karp integrator.

Comparing the density profiles in Fig. 7 (where the glass bead has been removed), instabilities in the form of filling factor fluctuations due to insufficient interaction numbers appear for smoothing lengths $h \leq 0.075 \mathrm{~mm}$, i.e., for $I_{\mathrm{av}} \leq 30$. For
Table 2. Parameters for the convergence study regarding interaction numbers.

\begin{tabular}{ccccccr}
\hline \hline$h$ & $h / l_{\mathrm{c}}$ & $I_{\min }$ & $I_{\mathrm{av}}$ & $I_{\max }$ & $T_{\text {comp }}$ & $N_{\text {steps }}$ \\
\hline $0.050 \mathrm{~mm}$ & 2 & 3 & 13 & 25 & $16.2 \mathrm{~h}$ & 132401 \\
$0.075 \mathrm{~mm}$ & 3 & 10 & 30 & 55 & $14.8 \mathrm{~h}$ & 96912 \\
$0.100 \mathrm{~mm}$ & 4 & 16 & 53 & 92 & $19.8 \mathrm{~h}$ & 71175 \\
$0.125 \mathrm{~mm}$ & 5 & 23 & 82 & 142 & $19.0 \mathrm{~h}$ & 56347 \\
$0.150 \mathrm{~mm}$ & 6 & 32 & 116 & 205 & $21.6 \mathrm{~h}$ & 46782 \\
$0.175 \mathrm{~mm}$ & 7 & 43 & 158 & 274 & $24.3 \mathrm{~h}$ & 39980 \\
\hline
\end{tabular}

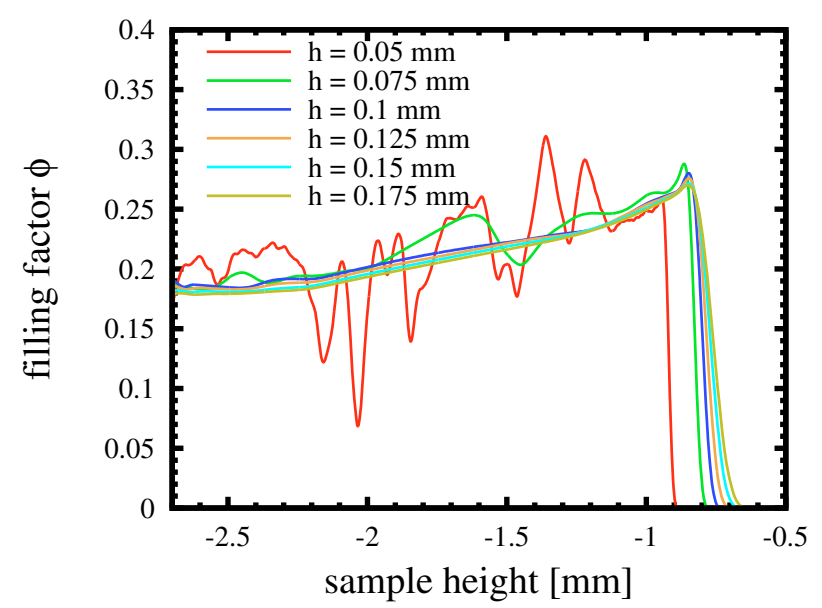

Fig. 7. Convergence study for the density profile using the 2D setup and varying the smoothing length $h$. Through this variation, the number of interaction partners is varied according to Table 2 . The glass bead has been removed in this plot. For $h \leq 0.075 \mathrm{~mm}$, clear signs of instabilities are visible. For $h \geq 0.1 \mathrm{~mm}$, the filling factor has the same value and its position remains constant. The smoothing of the boundary of the dust sample is increased for increasing $h$.

$h \geq 0.1 \mathrm{~mm}$, the density profile has essentially the same shape: the position and height of the filling factor peak remains nearly the same and $\phi$ drops smoothly to $\approx 0.18$ towards the bottom of the dust sample. Only the sharp edge at the top of the dust sample is smoothed across a wider range due to the increased smoothing length.

Table 2 shows that the number of integration steps $N_{\text {step }}$ decreases with increasing interaction numbers. This is because the elastic waves inside the dust sample are smoothed over a wider range causing the adaptive integrator to increase the duration of a time step, since density fluctuations do not have to be resolved as sharply as when a lower amount of smoothing is applied. As expected, the computation time $T_{\text {comp }}$ generally increases with the increasing number of interactions. There are two exceptions: $h=0.075 \mathrm{~mm}$ and $h=0.125 \mathrm{~mm}$. Here, the decrease in $N_{\text {steps }}$ overcompensates for the increase in the interactions leading to

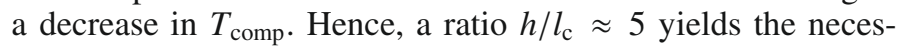
sary accuracy and an acceptable amount of computation time. This study also justifies the choice of $h / l_{\mathrm{c}}=5.6$ in Güttler et al. (2009) and we use this ratio throughout this paper.

According to these findings, for 3D simulations an average interaction number of theoretically $I_{\mathrm{av}}^{3 / 2} \approx 750$ would be needed to achieve the same numerical resolution. However, similar simulations are infeasible and our choice of $I_{\mathrm{av}} \approx 240$ in $3 \mathrm{D}$ is equivalent to $I_{\mathrm{av}} \approx 40$ in $2 \mathrm{D}$, which should provide sufficient and reliable accuracy. 


\subsection{Geometrical difference $-2 D$ and $3 D$ setups}

As one can easily see in Fig. 6, 2D and 3D simulations have significantly different convergence values for the intrusion depth. This deviation is caused by the geometrical difference of the $2 \mathrm{D}$ and 3D setup. The 2D setup (glass circle impacts into dust semicircle) represents a slice through a glass cylinder and a semicylindrical dust sample, which implies an infinite expansion into the third spatial direction. In contrast the 3D setup represents a real sphere dropping into a "bowl" of dust. The relation for the intrusion depth found by Güttler et al. (2009) (see Sect. 3) contains a geometrical dependence $D \propto m v / A$, where $D$ is the intrusion depth, $m$ is the mass of the impacting glass bead, $v$ is its impact velocity, $A$ is its cross-section, and $m_{2 \mathrm{D}}$ is the mass per unit length. Güttler et al. (2009) applied this relation to determine a rough correction factor between 2D and 3D simulation setups:

$\frac{m_{3 \mathrm{D}} v}{A_{3 \mathrm{D}}}=\frac{\frac{4}{3} \pi r^{3} \rho \cdot v}{\pi r^{2}}=\frac{8}{3 \pi} \frac{\pi r^{2} \rho \cdot v}{2 r}=\frac{8}{3 \pi} \frac{m_{2 \mathrm{D}} v}{A_{2 \mathrm{D}}}$.

Hence, the 2D intrusion depth has to be corrected by a factor of $\approx \frac{8}{3 \pi}$ to determine the 3D intrusion depth. The comparison is shown in Fig. 8. We again choose the 2D and 3D data gained in the convergence study for the peak filling factor values shown in Fig. 6. Figure 8 shows the original 2D data, the corrected 2D data, and the corresponding 3D data (with errorbars that indicate the smoothing length). The 3D values closely agree with the rough correction and remain well within the maximum error. This comparison justifies the correction of the results in Güttler et al. (2009). With the aid of this - now verified - correction factor, the $2 \mathrm{D}$ data of the intrusion depth can be converted into $3 \mathrm{D}$ data and all calibration tests involving the intrusion depth can be carried out in 2D, saving a significant amount of computation time. Comparing the vertical density profiles of $2 \mathrm{D}$ and $3 \mathrm{D}$ setups in Fig. 5 also resolves another unsolved issue in Güttler et al. (2009). According to the experimental data, the filling factor drops to a value of $\phi \approx 0.16$ within a distance of $\approx 0.6 \mathrm{~mm}$ from the bottom point of the glass bead. For high-resolution 2D simulations (Fig. 5, top), the filling factor does not drop to this value for the entire dust sample. However, the 3D simulations (Fig. 5, bottom) show that this is again because of the difference between the 2D and 3D geometry. With the 3D setup, the filling factor drops to $\phi \approx 0.16$ within $\approx 0.9 \mathrm{~mm}$. All deviations from experimental findings caused by this effect, in particular the large amount of volume with $\phi \leq 0.2$ in the cumulated volume over the filling factor diagram (Fig. 15 in Güttler et al. 2009), can in principal be removed by switching to 3D simulations. They do not represent a fundamental error in the porosity model.

\subsection{Artificial viscosity}

Since artificial viscosity plays an eminent role in the stability of SPH simulations, we investigate its influence on the outcome of our compaction calibration setup. Only artificial $\alpha$-viscosity is applied in our test case, but in a threefold way: (1) it dampens high oscillation modes of the glass bead caused by the stiff Murnaghan equation of state (EOS). Thereby it enlarges the time step of our adaptive integrator and saves computation time. (2) It is used to provide the dust material with a basic stability. (3) It separates the areas of Murnaghan EOS and dust EOS and prevents a so-called "cannonball instability". For all three cases, the influence of $\beta$-artificial viscosity was also tested, but its influence on all benchmark parameters was found to be negligible.

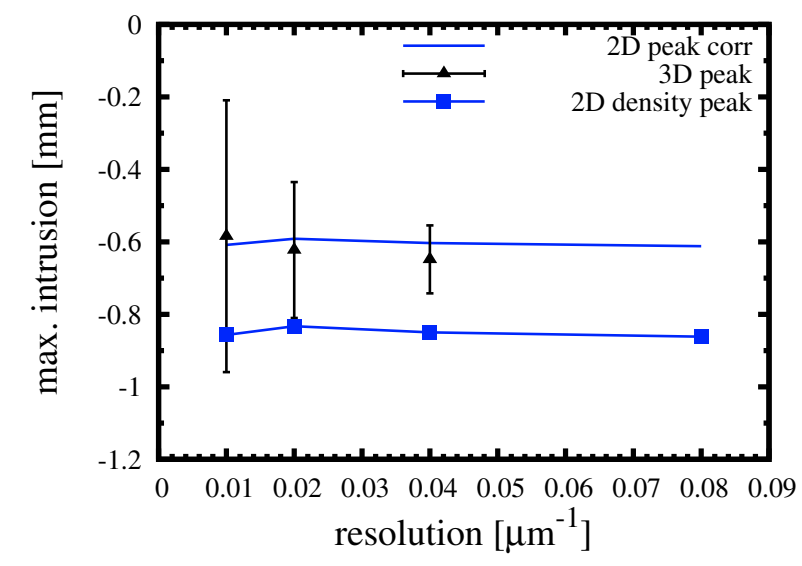

Fig. 8. Verification of the 2D-3D correction factor. Filled symbols denote the position of the filling factor peak of the dust material in Fig. 5. Triangles represent $3 \mathrm{D}$ and squares $2 \mathrm{D}$ values. The conversion from 2D to 3D intrusion depth utilising the correction factor from Eqs. (26) and (23) due to the geometrical difference is indicated by the line without symbols. The $3 \mathrm{D}$ values are in very good agreement with the very rough theoretical prediction. They lie well within the errors.

(1) The choice of the $\alpha$-viscosity of the glass bead proved to be unimportant. We choose the canonical value $\alpha=1.0$. No influence on the physical benchmark parameters was detected for all $\alpha$ values, except $\alpha=0$, which produces an instability. Values of $\alpha>1.0$ have no significant effect on the damping, and the influence for $0.1<\alpha<1.0$ is not too high, but still observable. Hence, we stick to the canonical value.

(2) Sirono (2004) applies no artificial viscosity to his porous ice material because of its spurious dissipative properties. Our findings, shown in Fig. 9, qualify this choice in terms of the dust material. Within our 2D compaction calibration setup $\left(l_{\mathrm{c}}=\right.$ $25 \mu \mathrm{m}, h / l_{\mathrm{c}}=5.6$ ), we vary $\alpha$ from 0 to 2 and observe its influence on the density profile (Fig. 9, top) and the maximum intrusion represented by the filling factor peak of the dust material (Fig. 9, bottom).

The position of the filling factor peak ranges from $\approx-0.92 \mathrm{~mm}$ with $\alpha=0.0$ to $\approx-0.62 \mathrm{~mm}$ at $\alpha=2.0$. This clearly demonstrates the dissipative feature of the $\alpha$-viscosity, since a lower amount of kinetic energy of the glass bead is transformed into plastic deformation with higher $\alpha$. The residual energy must have been dissipated. However, the $\alpha$-viscosity-intrusion curve seems to saturate at a value of $\approx-0.6 \mathrm{~mm}$. The decrease in the maximum intrusion can also be seen in the density profile (Fig. 9, top). While the profile maintains nearly the same shape, the height of the filling factor peak decreases with increasing $\alpha$. Hence, an increasing artificial viscosity diminishes the peak pressure during compaction by means of the compressive strength relation $\Sigma(\phi)$, which is directly responsible for the height of the filling factor peak.

In contrast to Sirono (2004), we find that it is necessary to apply a small amount of $\alpha$-viscosity to the dust material. For $\alpha<0.1$, the results show evidences of an instability, which is also responsible for a rapid increase in the maximum intrusion. Therefore, we find it convenient to apply an artificial viscosity with $\alpha=0.1$ to the dust material, which holds for the previous simulations of this section as well as the following. The choice of a non-zero $\alpha$, however, is also justified by experimental findings: after impacting into the dust sample, the glass bead shortly oscillates because of the elastic properties of the dust. This oscillation is damped by internal friction, which we model with artificial viscosity. Therefore, by choosing a non-zero $\alpha$ we take 
R. J. Geretshauser et al.: Numerical simulations of highly porous dust aggregates
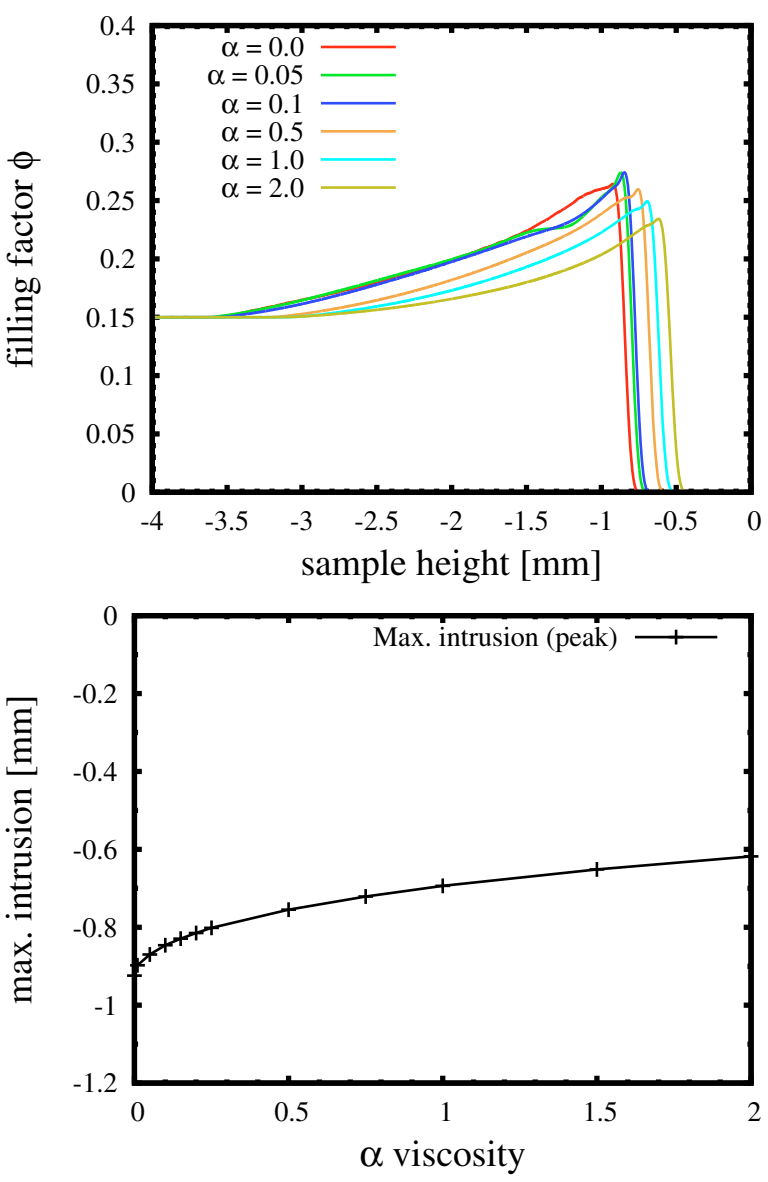

Fig. 9. Density profile (top) and maximum intrusion (bottom) for different values of artificial $\alpha$-viscosity. The shape of the density profile hardly changes, but increasing the $\alpha$-viscosity decreases both the maximum filling factor and the maximum intrusion depth.

into account the dissipative properties that our dust material naturally has. A quantitative calibration of this parameter, however, is left to future work.

(3) During our first simulations with the $2 \mathrm{D}$ compaction calibration setup, we observed what is sometimes described in the literature as "cannonball instability". During the compaction process, when the glass bead intrudes into the dust material, single particles at the sphere's surface begin to oscillate between the domains of the Murnaghan EOS and dust EOS. Because of the significant difference in the "stiffness" of these two equations of state, the particles acquire an enormous amount of kinetic energy until they move fast enough to generate a pressure on the dust material that exceeds the compressive strength $\Sigma(\phi)$. Eventually they disengage from the sphere's surface like a cannonball and dig themselves into the dust sample causing a huge amount of unphysical compaction. We tackle this problem for all SPH particles with dust EOS, which interact with glass bead SPH particles, by applying the same amount of $\alpha$-viscosity as for the sphere, i.e., $\alpha=1.0$. In our simulations, this is sufficient to prevent the "cannonball instability". The spurious dissipation caused by this measure is negligible.

\section{Calibration}

\subsection{Compressive strength and compaction properties}

We refine and extend a study of the compaction properties of the dust sample, which was already carried out in a similar, but less detailed way in Güttler et al. (2009). There the quantity having the strongest influence on the compaction was found to be the compressive strength relation $\Sigma(\phi)$ (Eq. (21)), which was measured in an omni-directional and static manner. To adapt this relation to dynamic compression, the mean pressure $p_{\mathrm{m}}$ and the "slope" of the Fermi-shaped curve $\Delta$ can be treated as free parameters. The upper and lower boundaries of the filling factor $\phi_{2}$ and $\phi_{1}$, respectively, remain constant even in the dynamic case. Güttler et al. (2009) found that by lowering $p_{\mathrm{m}}$, most of the features of the compaction calibration setup can be reproduced in a very satisfactory manner. As a result, the ratio of the filling factor to compressive strength curve (see also Fig. 2 in Güttler et al. 2009) is shifted towards lower pressures and the yield pressure for compression is lowered. Using only $2 \mathrm{D}$ simulations and a rough parameter grid, Güttler et al. (2009) fix $p_{\mathrm{m}}=1.3 \mathrm{kPa}$. The "slope" $\Delta$ has not been considered. In this work, we consider $\Delta$ and we perform more accurate parameter studies for $p_{\mathrm{m}}$. From the latter, we predict a reasonable choice for $p_{\mathrm{m}}$, which represents the basis of a 3D simulation of the compaction calibration setup. The results of this simulation are later compared to results from the laboratory. In this comparison, we use the same features as Güttler et al. (2009).

\subsubsection{Fixing free parameters}

Since no empirical data are available for $p_{\mathrm{m}}$ in the dynamical compressive strength curve, we perform a parameter study to determine a suitable choice for this important quantity. For this study, we use the 2D compaction calibration setup and vary $p_{\mathrm{m}}$ from 0.13 to $13.0 \mathrm{kPa}$ (Figs. 10 and 11 ), where $13.0 \mathrm{kPa}$ represents the value of the static compressive strength curve. The effect of lowering $p_{\mathrm{m}}$ can most clearly be seen in the vertical filling-factor profile (Fig. 10, top). First of all, more material can be compressed and is to higher filling factors. As a consequence, the glass bead intrudes more deeply into the dust sample. From experimental results, we expect an intrusion depth of about one sphere diameter $(\approx 1 \mathrm{~mm})$. With the aid of the empirical relation between the ratio of momentum to impactor cross-section $m v A^{-1}$ and intrusion depth $D$ (Eq. (23)) as well as the correction factor between 2D and 3D intrusion depth (Eq. (26)), we estimate that $D^{3 \mathrm{D}} \approx 1 \mathrm{~mm}$ corresponds to $D^{2 \mathrm{D}} \approx 1.42 \mathrm{~mm}$. Figure 11 shows the maximum intrusion over the stopping time for various values of $p_{\mathrm{m}}$ (labels). The estimated $D^{2 \mathrm{D}}$ is indicated by a dashed line. In terms of intrusion depth, we deduce that a dynamic mean pressure $p_{\mathrm{m}}=0.26 \mathrm{kPa}$ is a suitable choice.

This is supported by the peak filling factor appearing in the filling factor profile (Fig. 10, bottom). For the compaction calibration setup, empirical data indicate that a peak filling factor $\phi_{\max } \approx 0.3$ can be expected. The comparison between $2 \mathrm{D}$ and $3 \mathrm{D}$ results (Sect. 4.3) has shown that the peak filling factor in the vertical density profile in the $2 \mathrm{D}$ case is generally higher than for the same situation in $3 \mathrm{D}$. The equivalent of $\phi_{\max }^{3 \mathrm{D}} \approx 0.3$ is a maximum filling factor of $\phi_{\max }^{2 \mathrm{D}} \approx 0.34$ in $2 \mathrm{D}$. This points to a choice of $p_{\mathrm{m}} \approx 0.3 \mathrm{kPa}$, which is consistent with the findings for the intrusion depth.

The compressive strength relation $\Sigma(\phi)($ Eq. (21)) contains a second free parameter $\Delta$, which accounts for the "slope" of the Fermi-shaped curve. In Güttler et al. (2009), this parameter was chosen to be indentical to that of the static omni-directional compressive strength curve and a more careful investigation was not carried out. To understand its effect on the compaction properties of the dust sample, we utilise the $2 \mathrm{D}$ compaction calibration setup again and vary $\Delta$ from 0.55 to 0.80 . The results are presented in Fig. 12. From the vertical density profile (Fig. 12, top), 

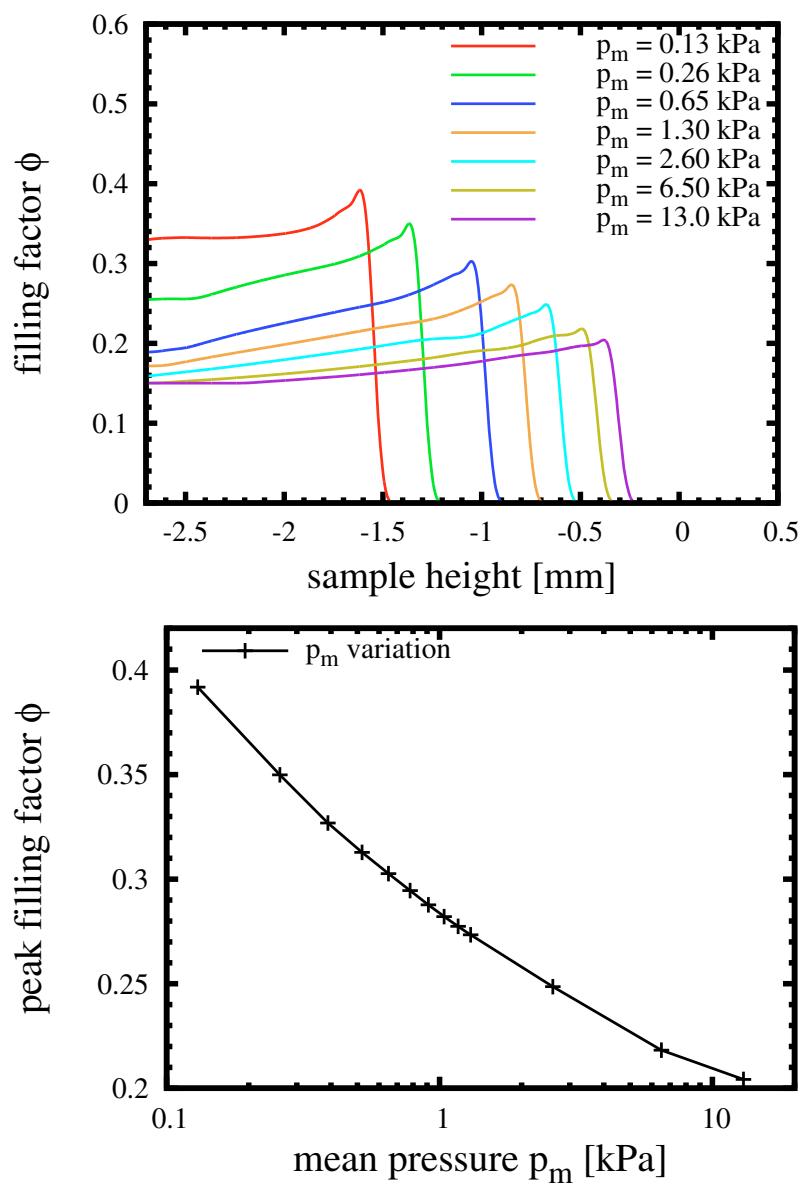

Fig. 10. Density profile (top) and maximum peak filling factor from the density profile over $p_{\mathrm{m}}$ (bottom) for different values of $p_{\mathrm{m}}$, which represents the mean pressure in the compressive strength relation $\Sigma(\phi)$ (Eq. (21)). Lowering $p_{\mathrm{m}}$ from $13.0 \mathrm{kPa}$ (static compressive strength) increases the compressed volume, its filling factor, and the maximum intrusion depth of the glass bead. The parameter study was performed using the $2 \mathrm{D}$ compaction calibration setup.

it can be seen that increasing $\Delta$ increases the intrusion depth, but not as effectively as lowering the mean pressure $p_{\mathrm{m}}$. This is because increasing $\Delta$ hardly increases the peak filling factor in the vertical density profile. More volume is compressed to lower filling factors. In contrast, by lowering $p_{\mathrm{m}}$ the total amount of volume compressed becomes smaller but is compacted to higher filling factors. This behaviour can be seen by comparing the density profiles for the $p_{\mathrm{m}}$ variation (Fig. 10, top) and $\Delta$ variation (Fig. 12, top) and in particular from the cumulated volume over filling factor curve (Fig. 12, bottom). This figure shows the normalised fraction of compacted volume corresponding to a volume filling factor $>\phi$. The intersection of the curves with the $y$-axis represents the total compressed volume, which is increased from $\approx 7$ to $\approx 9.5$ sphere volumes. The corresponding experimental measurements here infer a value of roughly one sphere volume (see Sect. 3.2). For $0.18<\phi<0.23$ in particular the compacted volume fraction is increased. The equivalent figure for the $p_{\mathrm{m}}$ variation can be found in Güttler et al. (2009, Fig. 15). By comparing the 2D and 3D calibration setups (Sect. 4.3), we infer that a significant amount of this compaction (especially in the lower filling factor regime) is caused by the geometrical difference. However, the experimental data do not indicate a particularly high amount of compaction to lower

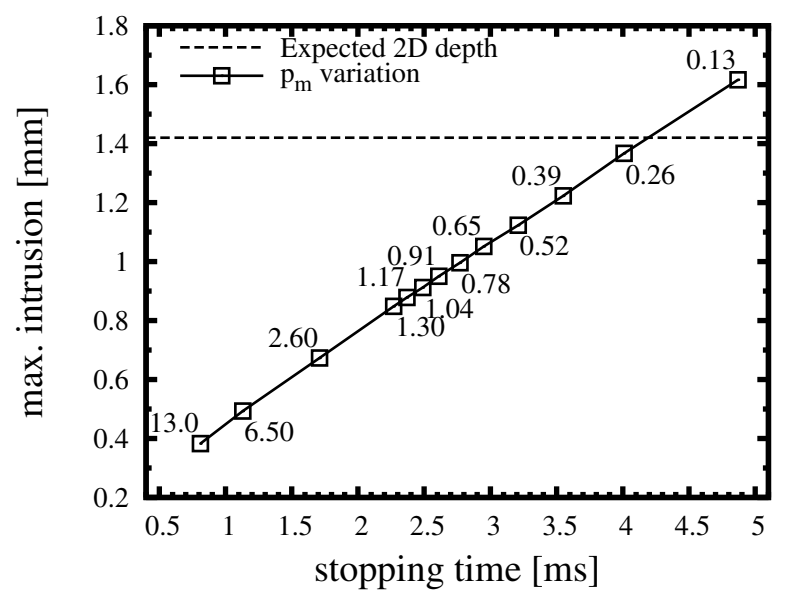

Fig. 11. Maximum intrusion over stopping time for different values of the mean pressure $p_{\mathrm{m}}$ (labels, in $\mathrm{kPa}$ ) in the compressive strength relation $\Sigma(\phi)$ (Eq. (21)) using the 2D compaction calibration setup. The dashed line indicates the 2D intrusion depth that is equivalent to a $3 \mathrm{D}$ intrusion depth of $\approx 1 \mathrm{~mm}$ according to Eq. (23) and the 2D-3D correction factor from Eq. (26). This supports the choice of the mean pressure $p_{\mathrm{m}}=0.26 \mathrm{kPa}$ for further simulations.
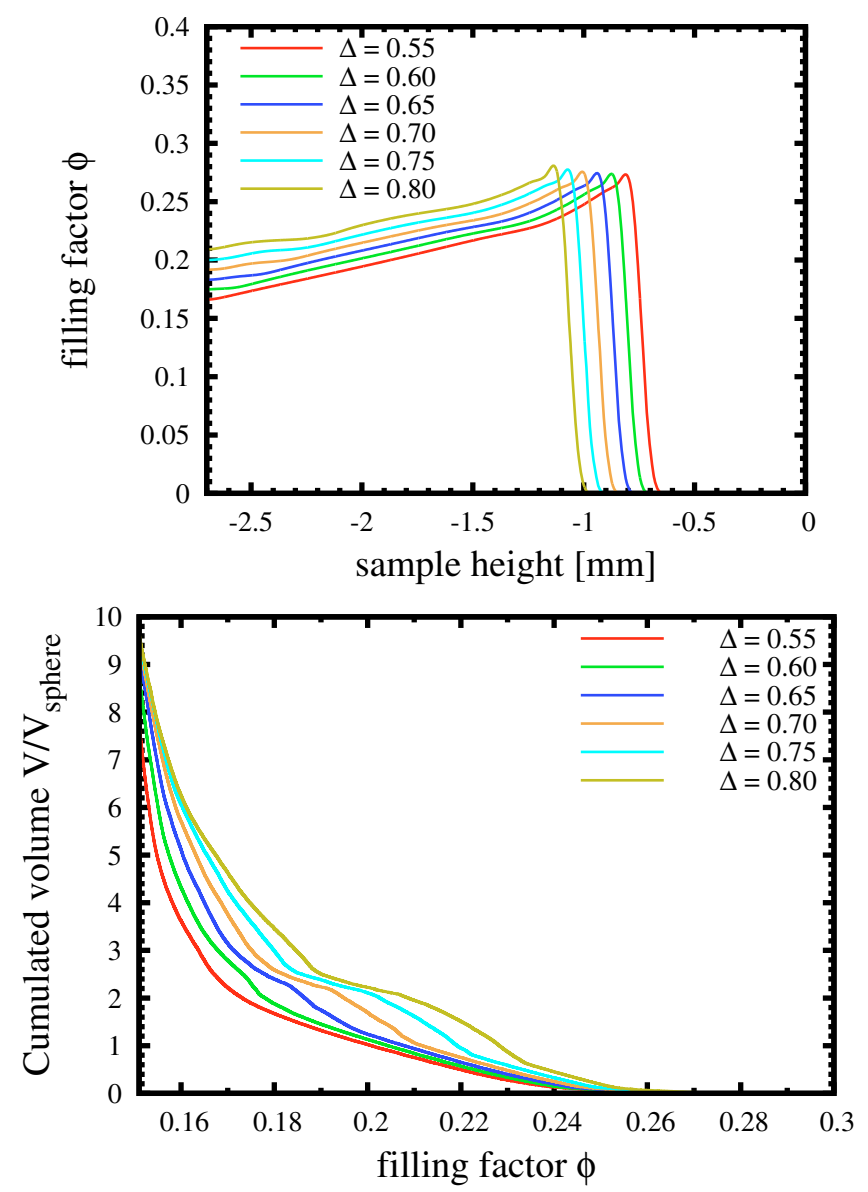

Fig. 12. Density profile (top) and normalised volume fraction of compacted volume over its corresponding filling factor (bottom) for different values of $\Delta$ in the compressive strength relation (Eq. (21)). Increasing $\Delta$ increases the amount of compacted volume at filling factors $0.18<\phi<0.23$ and thereby the maximum intrusion depth. In contrast, the peak filling factor in the density profile remains unchanged.

filling factors (rather the contrary) and, therefore, we maintain our initial choice of $\Delta=0.58$. 
R. J. Geretshauser et al.: Numerical simulations of highly porous dust aggregates

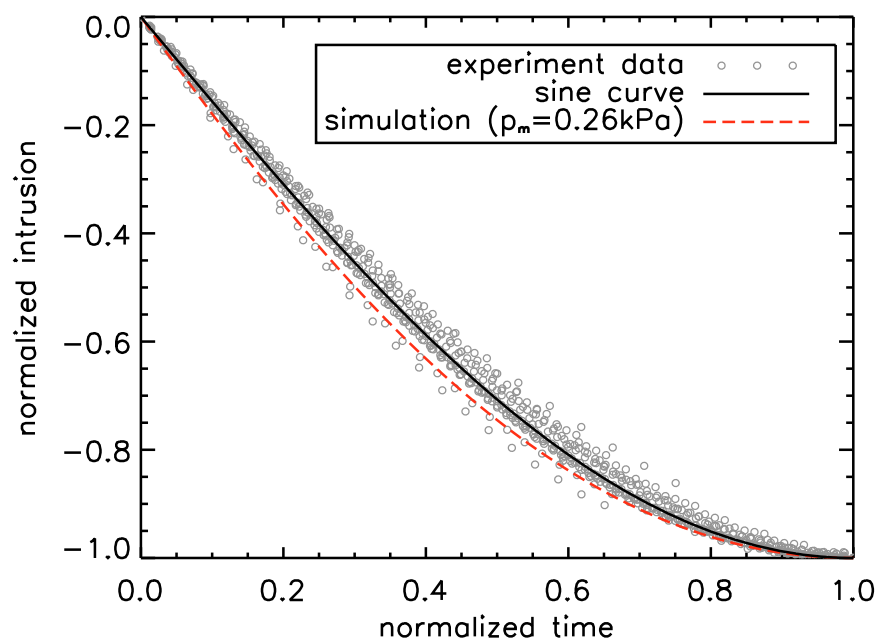

Fig. 13. Comparison between intrusion curves from drop experiments using $1 \mathrm{~mm}$ and $3 \mathrm{~mm}$ spheres and our 3D simulation (mean pressure $p_{\mathrm{m}}=0.26 \mathrm{kPa}$ ). The curves are normalised such that the origin represents first touch of sphere and dust sample and $(1,-1)$ denotes stopping time at maximum intrusion. The simulated curve lies slightly underneath the fit to the experimental data, but well within the errors. The deviation is due to a shorter stopping time than in the experiments.

\subsubsection{Comparison with experiments}

To compare with experiments, we performed a simulation using the 3D compaction calibration setup (see Table 1) with two exceptions: (1) the bulk modulus of the dust material was set to $K_{0}=2 \mathrm{kPa}$ (instead of $K_{0}=300 \mathrm{kPa}$ ) since findings presented below indicated a much lower bulk modulus. However, the choice of $K_{0}$ has little influence on the compaction properties calibrated for in the compaction calibration setup. It is more important for bouncing and fragmentation, as shown below. (2) The mean pressure of the compressive strength relation (Eq. (21)) was set to $p_{\mathrm{m}}=0.26 \mathrm{kPa}$ as suggested in the previous section.

The following features of the compaction calibration setup were measured in the laboratory and are used here for comparison: (1) the stopping time $T_{\mathrm{s}}$; (2) the intrusion curve of the projectile; (3) the vertical density profile; (4) the cumulated volume over filling factor curve; and (5) the cross-section through both the sphere and the dust sample displaying the filling factor.

(1) The experiments show that the stopping time $T_{\mathrm{s}}$ of the glass bead, i.e., the time from first contact with the dust sample until deepest intrusion, is nearly constant at $T_{\mathrm{s}}^{\exp }=3.0 \pm$ $0.1 \mathrm{~ms}$ for $1 \mathrm{~mm}$ projectiles over different impact velocities (see Sect. 3.1). In our simulation, we find that $T_{\mathrm{s}}^{\mathrm{sim}}=2.42 \pm$ $0.05 \mathrm{~ms}$, which is not in excellent agreement but also not too far off the experimental results.

(2) The intrusion curve $h(t)$ was cleared from gravity effects and normalised by evaluating $h^{\prime}\left(t^{\prime}\right)=h(t) / D$ and $t^{\prime}=t / T_{S}$, where $h(t)$ is the position of the bottom of the glass bead as a function of time, $D$ the maximum intrusion depth, and $T_{\mathrm{s}}$ the stopping time. At first contact, $t$ is given by $h^{\prime}\left(t^{\prime}=0\right)=0$ and at deepest intrusion $h^{\prime}\left(t^{\prime}=1\right)=-1$ (see also Güttler et al. 2009, Sect. 3.2.2). The comparison is shown in Fig. 13: the intrusion curve generated by our simulation lies well within the data from the experiments with $1 \mathrm{~mm}$ and $3 \mathrm{~mm}$ spheres and only slightly below the sine curve fitted to the empirical data.

(3) The comparison between the experimentally measured vertical density profile and the results of our simulation is shown in Fig. 14. The crosses represent the data from two experiments

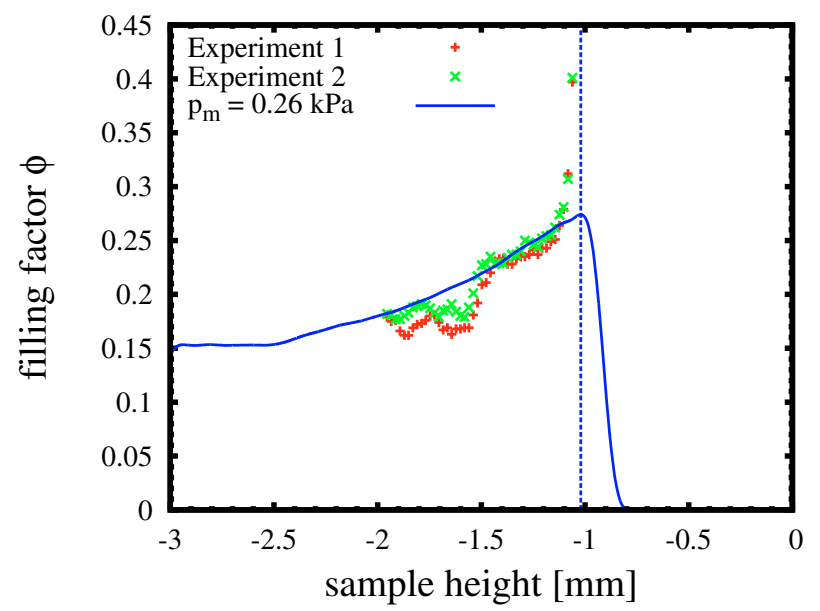

Fig. 14. Comparison between experimentally measured (crosses, sphere not removed) and simulated (solid line, sphere removed) density profiles at maximum intrusion for the compaction calibration setup. The dashed line indicates the position of the simulated maximum intrusion depth given by the density peak at $-1.02 \mathrm{~mm}$. The simulation was carried out in $3 \mathrm{D}$ using a mean pressure $p_{\mathrm{m}}=0.26 \mathrm{kPa}$ for the compressive strength relation (Eq. (21)). Both profiles are in excellent agreement. That the step-like structure of the experimental data cannot be seen in the simulation is a minor drawback since it is clearly interpolated.

in which the sphere was not removed during measurement. For this reason, the filling factor reaches extremely high values at $\approx-1 \mathrm{~mm}$, which corresponds to the bottom of the glass bead. The vertical density profile of our simulation is given by the solid line and the vertical dashed line is placed at its filling factor peak at $-1.02 \mathrm{~mm}$ representing the maximum intrusion depth. Compared to the experimentally measured maximum intrusion depth of $-1.07 \mathrm{~mm}$, this is an excellent result. Since a depth of $\approx-1 \mathrm{~mm}$ was required using Eqs. (23) and (26) and the 2D intrusion depth study of the previous section, this result also supports the validity of these relations. In addition to the exact value of the intrusion depth, our simulation also reproduces the shape of the given experimental vertical density profile very well. Only the step-like structure at $\approx-1.5 \mathrm{~mm}$ is not reproduced by our simulation but is instead interpolated.

(4) The vertical density profile shows only a cut through the compressed volume. It contains information about the exact structure of the compression. The cumulated volume over filling factor curve (Fig. 15) has the advantage of representing the total compressed volume and its filling factors, hence, these features are not fully independent but focus on different aspects of the compression. The cumulated volume is normalised by the sphere volume to account for the volume fraction of the compressed area that corresponds to a volume filling factor $>\phi$. In general, the experimental reference and our simulation show close agreement. A slightly too large volume is compressed to high filling factors in the simulation, which leads to an almost constant deviation for $\phi<0.26$. However, the slope is reproduced very well.

(5) The comparison between the cross-sections through both the sphere and dust sample along the $z$-axis (Fig. 16) indicates where there is an excess of compressed volume. First, the crosssection of the sphere is artificially enhanced by the smoothing of its boundaries, which is inherent to the SPH method. One effect of the smoothing is the existence of a gap between the sphere and dust sample, as already discussed in Sect. 4.2 and clearly visible in Fig. 16 (top). Hence, we assume that the dust sample actually begins where it experiences its maximum compression. The sphere pokes out of the dust sample a bit more than in the 


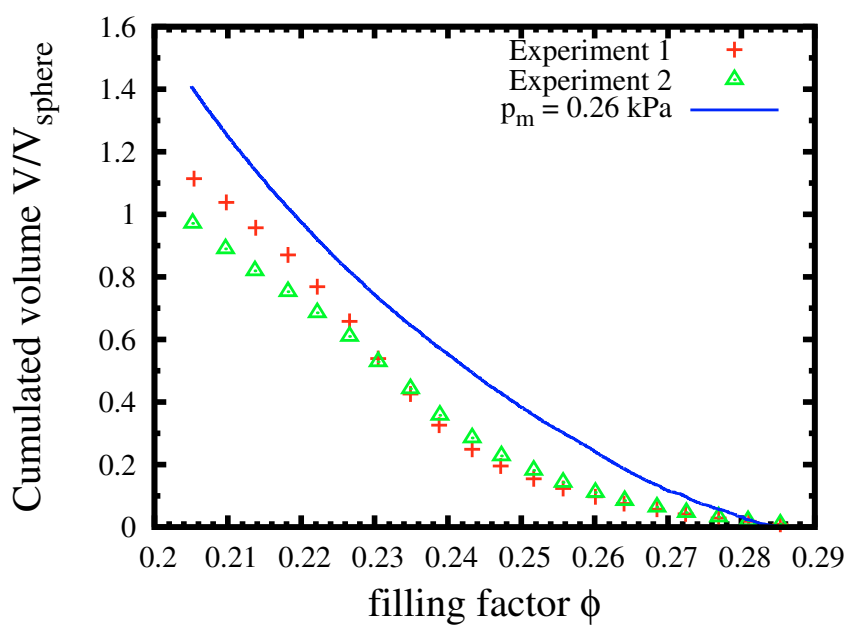

Fig. 15. Total cumulated volume over filling factor for drop experiments (crosses and triangles) and our 3D simulation with mean pressure $p_{\mathrm{m}}=0.26 \mathrm{kPa}$ (solid line). The plot shows the cumulated mass fraction (normalised by the sphere volume) with a filling factor $>\phi$ over $\phi$. Our simulation is in good agreement with the experimental findings. However, a greater amount of volume being compressed to high filling factors leads to an almost constant deviation for $\phi<0.26$. The slope is reproduced very well.

experiment because of the artificial enlargement of the crosssection. Second, it can be seen that in the experimental reference (Fig. 16, bottom) the compressed region is much narrower and more concentrated beneath the sphere. In the simulated result, the compacted region is a bit broader. This indicates that the shear strength seems to be slightly lower than we assume. Third, the compression extends to too high filling factors, as was already visible in the cumulated volume diagram. Nevertheless, both cross-sections match very well, especially with respect to the mediocre resolution. Remarkably, even the slight intrusion channel on the left and right side of the sphere, which features a slight compression, can be reproduced.

\subsection{Bulk modulus}

As mentioned in Sect. 3.1, there are two estimates of the bulk modulus $K_{0}$ for the uncompressed material with $\phi \approx 0.15$. In our model, $K_{0}$ is also the pre-factor for computing the bulk modulus of higher filling factors with the aid of a power law (Eq. (9)). Since these indirect findings, i.e., $1 \mathrm{kPa}$ (see Weidling et al. 2009) and $300 \mathrm{kPa}$ (from sound speed measured by Blum \& Wurm 2008; Paszun \& Dominik 2008), differ by two orders of magnitude, we try to find a suitable value for $K_{0}$ utilising a calibration experiment.

Simulating the low velocity collision setup by Weidling et al. (2009), a 3D dust sphere $\left(\phi_{i}=0.15,1 \mathrm{~mm}\right.$ diameter, 267737 particles, $\left.l_{\mathrm{c}}=12.5 \mu \mathrm{m}\right)$ drops onto a solid surface (cylindrical, $1.6 \mathrm{~mm}$ diameter, $0.1 \mathrm{~mm}$ thick, 115677 particles, $\left.l_{\mathrm{c}}=12.5 \mu \mathrm{m}\right)$ with initial velocity $v_{0}=0.2 \mathrm{~m} \mathrm{~s}^{-1}$ (see Fig. 17). The material parameters are shown in Table 3. The bulk modulus $K_{0}$ is varied with respect to two values of the mean pressure $p_{\mathrm{m}}$ in the compressive strength relation $(0.26 \mathrm{kPa}$ and $1.3 \mathrm{kPa}$ ). During the impact, a small region of the bottom of the dust sphere is compressed. The deformed sphere then bounces off the target with reduced velocity $v_{\mathrm{f}}$ (see Fig. 17). The latter effect was already observed in Güttler et al. (2009) and demonstrates the ability of our code and the implemented porosity model to simulate the elastic properties of the dust correctly.
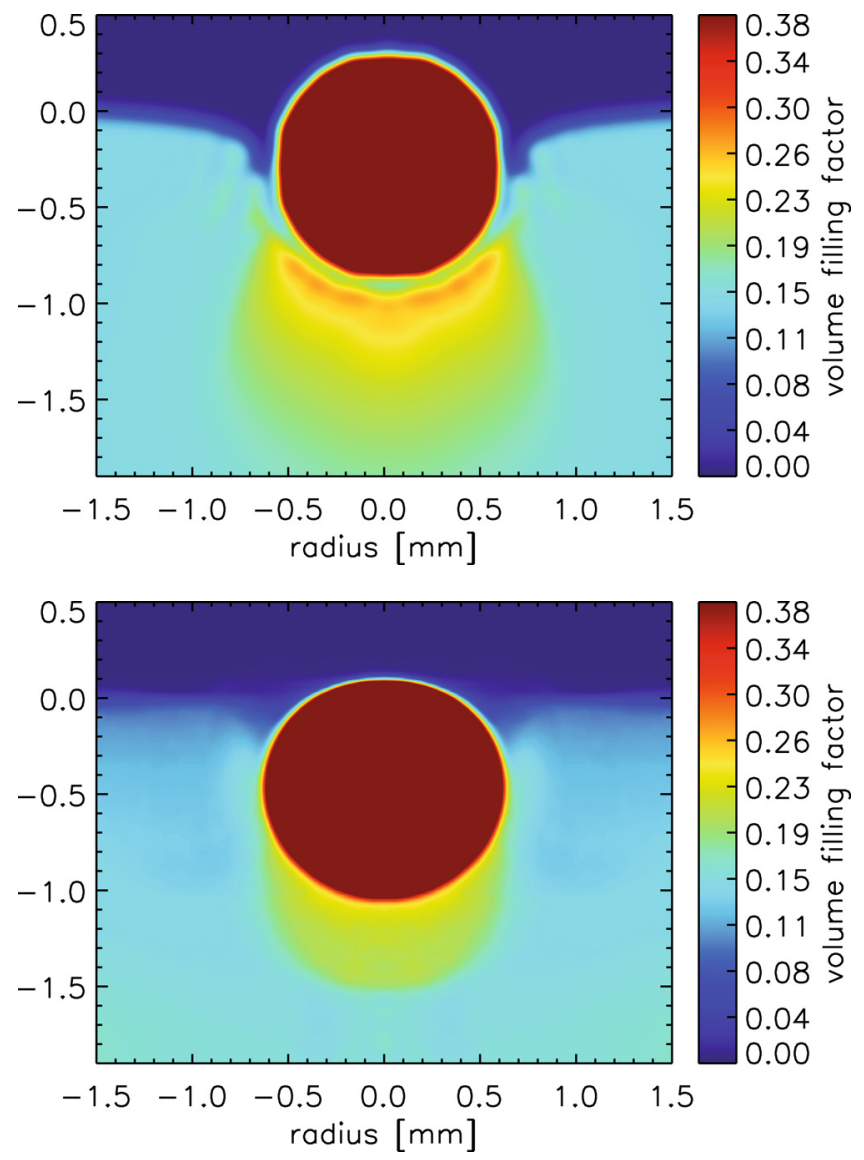

Fig. 16. Cross-section through glass bead (red) and dust sample (light blue) at maximum intrusion for our 3D simulation (with $p_{\mathrm{m}}=0.26 \mathrm{kPa}$, top) and the drop experiment (bottom). The colour indicates the spatially averaged filling factor. The density structures beneath the glass bead match very well. Even the slight compression along the tight intrusion channel can be reproduced. In the simulated plot, a gap between glass bead and the most dense area is clearly visible. This is caused by the smoothing of the sphere and was discussed in Sect. 4.2. The gap has roughly the size of one smoothing length $h$.

In this study, we doubled the resolution and determined the coefficient of restitution $\varepsilon_{\text {rest }}=v_{\mathrm{f}} v_{0}^{-1}$ depending on $K_{0}$ (see Fig. 18).

The bouncing calibration setup is equivalent with two centrally colliding dust aggregates at $0.4 \mathrm{~m} \mathrm{~s}^{-1}$, thus, our results are comparable to Heißelmann et al. (2007): $\varepsilon_{\text {rest }} \approx 0.2$ and $\approx 95 \%$ energy dissipation.

Based on the results of the previous section, where $p_{\mathrm{m}}=$ $0.26 \mathrm{kPa}$ turned out to be a good choice of the mean pressure, our results for this experiment favour a bulk modulus $K_{0} \approx$ $5 \mathrm{kPa}$. This value is close to the value $K_{0}=1 \mathrm{kPa}$ computed by Weidling et al. (2009) with a simplified model. Our simulations yield a coefficient of restitution $\varepsilon_{\text {rest }}=0.19(\approx 96 \%$ energy dissipation) for $K_{0}=5.0 \mathrm{kPa}$, which is in excellent agreement with the experimental results. On the other hand, for $K_{0}=500 \mathrm{kPa}$ we find $\varepsilon_{\text {rest }}=0.09(\approx 99 \%$ energy dissipation $)$, which is too far away from the reference value. A high value for the bulk modulus $K_{0}$ as given by the sound speed measurements is therefore excluded.

Given the higher value $p_{\mathrm{m}}=1.3 \mathrm{kPa}$ for the compressive strength curve, $\varepsilon_{\text {rest }}$ increases for all choices of $K_{0}$. For $K_{0}=$ $1.0 \mathrm{kPa}$, it becomes $\varepsilon_{\text {rest }} \approx 0.7$ and only $\approx 50 \%$ of the energy is dissipated. On the other hand, for $K_{0}=300 \mathrm{kPa}$ we find that $\varepsilon_{\text {rest }} \approx 0.13$, which is equivalent to $\approx 98 \%$ energy dissipation. 


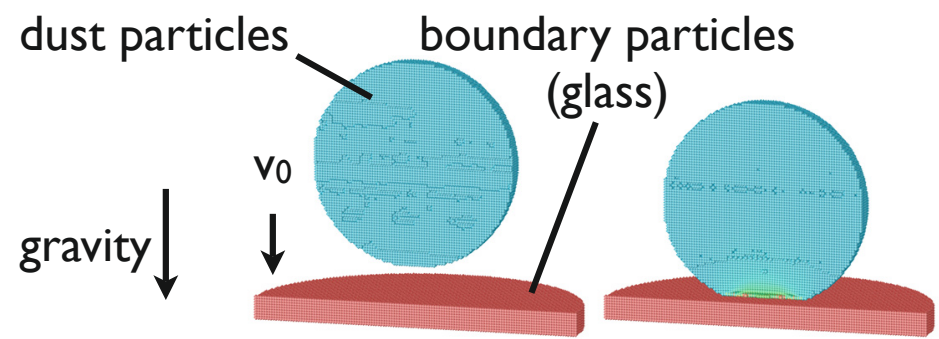

(a)

(b)

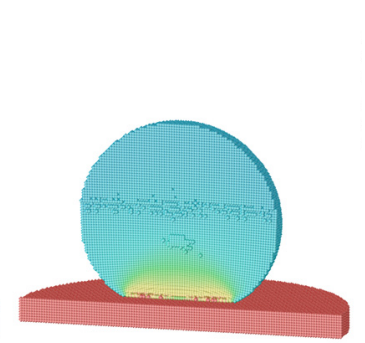

(c)

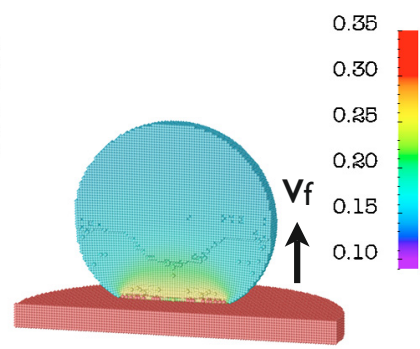

(d)

Fig. 17. Bouncing sequence for $t=0 \mathrm{~ms} \mathbf{a}), t=10 \mathrm{~ms} \mathbf{b}), t=18 \mathrm{~ms} \mathbf{c}$ ), and $t=25 \mathrm{~ms} \mathbf{d})$. The colour code indicates the filling factor. An aggregate consisting of dust particles (Sirono EOS, diameter $1.0 \mathrm{~mm}$ ) hits a solid surface simulated by boundary glass particles (Murnaghan EOS, diameter $1.6 \mathrm{~mm}$, thickness $0.1 \mathrm{~mm}$ ) with a velocity of $v_{0}=0.2 \mathrm{~m} \mathrm{~s}^{-1}$. For this simulation a bulk modulus of $K_{0}=5.0 \mathrm{kPa}$ and $p_{\mathrm{m}}=0.26 \mathrm{kPa}$ were used. The aggregate hits the surface and starts to be compacted at its bottom b). While the plastic deformation at the bottom increases, the aggregate is also deformed elastically: it gets broader c). Eventually it leaves the surface with a final velocity $v_{\mathrm{f}} \mathbf{d}$ ). It features a permanent compaction, while the elastic deformation vanishes. (An animation of this figure is available in the online journal.)

Table 3. Numerical parameters for the bouncing calibration setup.

\begin{tabular}{lccl}
\hline \hline Physical quantity & Symbol & Value & Unit \\
\hline Glass plate & & & \\
Bulk density $^{(*)}$ & $\rho_{0}$ & 2540 & $\mathrm{~kg} \mathrm{~m}^{-3}$ \\
Bulk modulus $^{(*)}$ & $K_{0}$ & $5 \times 10^{9}$ & $\mathrm{~Pa}$ \\
Murnaghan exponent $^{(*)}$ & $n$ & 4 & - \\
Radius & $r_{\text {plate }}$ & $0.8 \times 10^{-3}$ & $\mathrm{~m}$ \\
Thickness & $d_{\text {plate }}$ & $0.1 \times 10^{-3}$ & $\mathrm{~m}$ \\
\hline Dust sample & & & \\
Initial density & $\rho_{i}$ & 300 & $\mathrm{~kg} \mathrm{~m}^{-3}$ \\
Bulk density & $\rho_{\mathrm{s}}$ & 2000 & $\mathrm{~kg} \mathrm{~m}^{-3}$ \\
Reference density & $\rho_{0}^{\prime}$ & 300 & $\mathrm{~kg} \mathrm{~m}^{-3}$ \\
Initial filling factor & $\phi_{i}$ & 0.15 & - \\
Bulk modulus & $K_{0}$ & various & $\mathrm{Pa}^{\prime}$ \\
ODC mean pressure & $p_{\mathrm{m}}$ & 260 and 1300 & $\mathrm{~Pa}^{-}$ \\
ODC max. filling factor & $\phi_{2}$ & 0.58 & - \\
ODC min. filling factor & $\phi_{1}$ & 0.12 & - \\
ODC slope & $\Delta$ & 0.58 & - \\
Impact velocity & $v_{0}$ & 0.2 & $\mathrm{~m} \mathrm{~s}^{-1}$ \\
Radius & $r$ & $0.5 \times 10^{-3}$ & $\mathrm{~m}$ \\
\hline
\end{tabular}

Notes. ODC stands for omni-directional compression relation (Eq. (21)). Quantities marked by $(*)$ represent the parameters for sandstone in Melosh (1989), which we adopt for glass here.

For $p_{\mathrm{m}}=1.3 \mathrm{kPa}$, it is harder to dissipate energy by plastic deformation. Hence, less energy is dissipated in the compaction process and the coefficient of restitution is generally higher.

This bouncing experiment fixes our choice of the bulk modulus to $K_{0} \approx 5 \mathrm{kPa}$ while maintaining $p_{\mathrm{m}}=0.26 \mathrm{kPa}$ derived from the compaction experiment in the previous section. In the next section, we show that this choice is also consistent with the fragmentation behaviour of the dust aggregates.

\subsection{Fragmentation}

Since the intended field of application of our calibrated SPH code is the simulation of pre-planetesimal collisions, it is of major importance to calibrate and test the fragmentation behaviour of the simulated material. For this reason, we simulate the fragmentation experiment described in the second part of Sect. 3.3.

In our simulation, a dust aggregate $\left(\phi_{i}=0.35\right.$, 189296 particles) hits a glass plate (188478 particles) from below with an impact velocity of $v_{0}=8.4 \mathrm{~m} \mathrm{~s}^{-1}$. The spatial

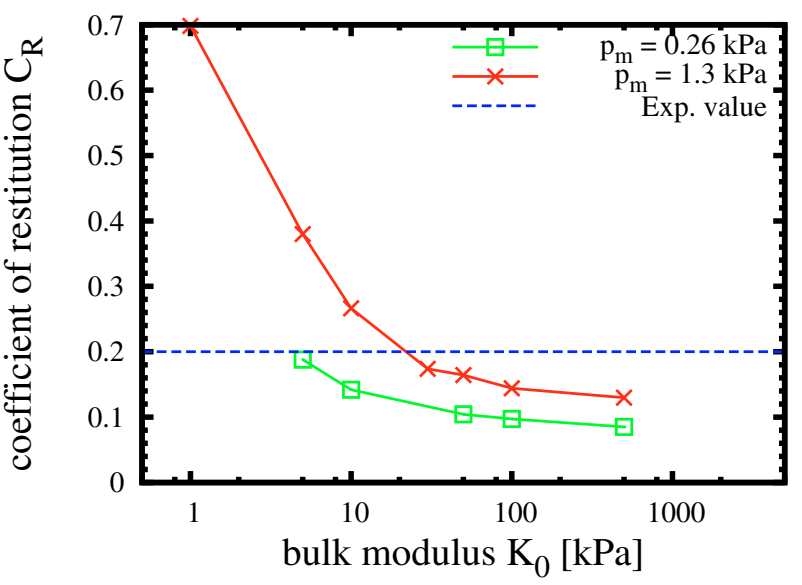

Fig. 18. Coefficient of restitution $\varepsilon_{\text {rest }}$ over bulk modulus $K_{0}$ for values of the mean pressure $p_{\mathrm{m}}$ of the compressive strength relation. For the lower values of $p_{\mathrm{m}}=0.26 \mathrm{kPa}$ (squares), more energy is dissipated by plastic deformation. For this reason, the coefficient of restitution is significantly lower than for the higher $p_{\mathrm{m}}=1.3 \mathrm{kPa}$ (crosses). Closest agreement with the experimental reference of $\varepsilon_{\text {rest }}=0.2$ (dashed line) is found for $K_{0} \approx 5 \mathrm{kPa}$ and $K_{0} \approx 20 \mathrm{kPa}$, respectively.

resolution $\left(l_{\mathrm{c}}=8.0 \mu \mathrm{m}, h=30.0 \mu \mathrm{m}\right)$ was chosen such that a single particle has less than $5 \times 10^{-6}$ times the mass of the whole aggregate $\left(6.8 \times 10^{-8} \mathrm{~kg}\right)$ to be able to resolve the same fragment masses as the experimental reference. Gravitation was taken into account. A list of the other relevant parameters can be found in Table 4 .

We investigated the influence of the bulk modulus on the fragmentation behaviour. For this reason, we varied $K_{0}$ from $3.0 \mathrm{kPa}$ to $6.5 \mathrm{kPa}$. For comparison with the reference experiment (Fig. 2), we also plot our fragmentation data in a cumulative way (Fig. 19) and find a good agreement with the powerlaw distribution of Eq. (24). The simulation was evaluated after $0.8 \mathrm{~ms}$ of simulated time. The mass of a fragment is given by the sum of the mass of the SPH particles belonging to it. We consider two fragments as being separated when they are not linked by SPH particles that interact with each other, i.e., when the closest SPH particles of two fragments are separated by a distance of greater than a smoothing length.

Güttler et al. (2009) faced the problem that when using $K_{0}=300 \mathrm{kPa}$ almost no fragmentation occurred (see Fig. 21 in this reference). Their speculations about a modification of the 
Table 4. Numerical parameters for the fragmentation calibration setup.

\begin{tabular}{lccl}
\hline \hline Physical quantity & Symbol & Value & Unit \\
\hline Glass plate & & & \\
Bulk density $^{(*)}$ & $\rho_{0}$ & 2540 & $\mathrm{~kg} \mathrm{~m}^{-3}$ \\
Bulk modulus $^{(*)}$ & $K_{0}$ & $5 \times 10^{9}$ & $\mathrm{~Pa}$ \\
Murnaghan exponent $^{(*)}$ & $n$ & 4 & - \\
Radius & $r_{\text {plate }}$ & $0.8 \times 10^{-3}$ & $\mathrm{~m}$ \\
Thickness & $d_{\text {plate }}$ & $0.04 \times 10^{-3}$ & $\mathrm{~m}$ \\
\hline Dust sample & & & \\
Initial density & $\rho_{i}$ & 300 & $\mathrm{~kg} \mathrm{~m}^{-3}$ \\
Bulk density & $\rho_{\mathrm{s}}$ & 2000 & $\mathrm{~kg} \mathrm{~m}^{-3}$ \\
Reference density & $\rho_{0}^{\prime}$ & 700 & $\mathrm{~kg} \mathrm{~m}^{-3}$ \\
Initial filling factor & $\phi_{i}$ & 0.35 & - \\
Bulk modulus & $K_{0}$ & various & $\mathrm{Pa}^{\prime}$ \\
ODC mean pressure & $p_{\mathrm{m}}$ & 260 & $\mathrm{~Pa}^{\prime}$ \\
ODC max. filling factor & $\phi_{2}$ & 0.58 & - \\
ODC min. filling factor & $\phi_{1}$ & 0.12 & - \\
ODC slope & $\Delta$ & 0.58 & - \\
Impact velocity & $v_{0}$ & 8.4 & $\mathrm{~m} \mathrm{~s}^{-1}$ \\
Radius & $r$ & $0.285 \times 10^{-3}$ & $\mathrm{~m}^{-}$ \\
\hline
\end{tabular}

Notes. ODC stands for omni-directional compression relation (Eq. (21)). Quantities marked by $(*)$ represent the parameters for sandstone in Melosh (1989), which we adopt for glass here.

shear strength to resolve this problem proved to be wrong. The quantity with the most impact on the fragmentation behaviour of the dust aggregate is its bulk modulus. In general, it can be said that an increase in the bulk modulus leads to an increase in the slope $\alpha$ of the fragment distribution, whereas the size of the largest fragment (normalised through the total mass of the projectile) $\mu$ remains roughly constant at $\approx 20 \%$ up to $K_{0}=6.0 \mathrm{kPa}$. For higher $K_{0}$, small chunks and single SPH particles mainly originate in the aggregate, which is only compressed but does not fragment. This reproduces the situation described in Güttler et al. (2009) and Sect. 3.

We now calibrate $\alpha$, which is more sensitive to changes in $K_{0}$ (see Table 5). Given the measured value of $\alpha=0.67$, we find excellent agreement with our simulation results using $K_{0}=4.5 \mathrm{kPa}$, which yields $\alpha=0.673 \pm 0.017$. This simulation also reproduces the experimentally measured normalised mass of the largest fragment $\mu=0.22$ to very high accuracy ( $\mu=0.234 \pm 0.007)$. The slight overestimation may be caused by the increase in the filling factor not being taken into account in the analysis of the experimental data (see Sect. 3), whereas in the simulation it is. The fragment distributions for different $K_{0}$ and the best fit for the power-law are shown in Fig. 19. The Setup and outcome of the simulation are displayed in Fig. 20. As already indicated in Sect. 5.2 the choice of $p_{\mathrm{m}}=0.26 \mathrm{kPa}$ and $K_{0}=4.5 \mathrm{kPa}$ is consistent with the results of the compression and bouncing experiments. The fragmentation experiment proves of the validity of these choices and the consistency of the underlying porosity model.

In contrast to the experiments, we find that no material sticks to the glass plate because of the setup of the simulation. As in Sect. 4.4, we use artificial viscosity to separate the glass and dust materials. This leads to an additional pressure on the dust material, which prevents sticking.

\section{Discussion}

We have presented a smooth particle hydrodynamics (SPH) code equipped with extensions for continuum mechanics of solid

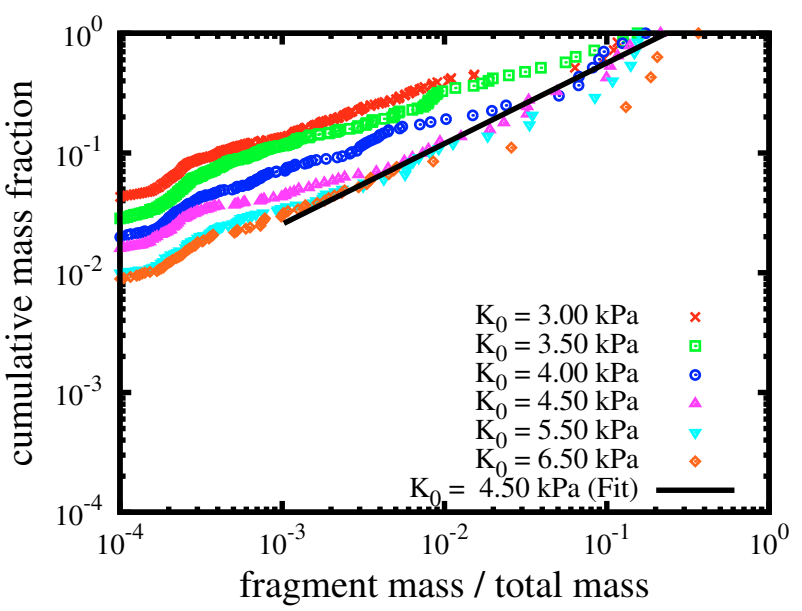

Fig. 19. Cumulative mass distribution of the fragments of a dust aggregate impacting on a glass plate for different values of $K_{0}$. For low fragment masses the shape of all simulated curves differs from the experimental curve in Fig. 2 due to the limited resolution of the experimental setup. An increase of $K_{0}$ leads to an increase in the slope $\alpha$ of the power-law fit. The best agreement with the experimentally measured slope 0.67 was found for the simulation with $K_{0}=4.5 \mathrm{kPa}$.

Table 5. Results from the fragmentation calibration setup.

\begin{tabular}{ccc}
\hline \hline$K_{0}[\mathrm{kPa}]$ & Slope $\alpha$ & Norm. largest fragment $\mu$ \\
\hline 3.00 & $0.361 \pm 0.004$ & $0.200 \pm 0.008$ \\
3.50 & $0.429 \pm 0.002$ & $0.172 \pm 0.002$ \\
4.00 & $0.518 \pm 0.011$ & $0.230 \pm 0.009$ \\
4.25 & $0.523 \pm 0.006$ & $0.194 \pm 0.004$ \\
4.50 & $0.673 \pm 0.017$ & $0.234 \pm 0.007$ \\
4.75 & $0.834 \pm 0.025$ & $0.196 \pm 0.005$ \\
5.00 & $0.832 \pm 0.063$ & $0.198 \pm 0.011$ \\
5.50 & $0.836 \pm 0.052$ & $0.220 \pm 0.010$ \\
6.00 & $2.027 \pm 0.121$ & $0.171 \pm 0.002$ \\
6.50 & $0.910 \pm 0.053$ & $0.390 \pm 0.013$ \\
\hline
\end{tabular}

Notes. The slope $\alpha$ of the power law increases with increasing bulk modulus $K_{0}$. Remarkably, the size of the normalised biggest fragment remains nearly constant around $\mu \approx 0.2$ for $K_{0} \leq 6.0 \mathrm{kPa}$.

bodies and an extended version of the Sirono (2004) porosity model. The code uses experimentally measured macroscopic parameters such as tensile strength and a static compressive strength relation. In Güttler et al. (2009), this code was used to determine a relation for the shear strength and to estimate the mean pressure $p_{\mathrm{m}}$ for the dynamic compressive strength relation (Eq. (21)). The estimate, however, made many assumptions because of the usage of 2D simulations only.

This work has profoundly investigated the numerical properties of the SPH code. Utilising the compaction calibration setup of Güttler et al. (2009) as an example, we have determined the adequate size of the computational domain and the adequate numerical and spatial resolutions. We have shown that the results for this setup converge at higher spatial resolutions. Boundary conditions are necessary for all calibration setups presented in this work. Their treatment, which is a difficult issue in SPH, was resolved by using boundary particles with vanishing acceleration at every time step. The dissipative properties of the artificial viscosity and its role for the stability of the simulation were investigated. Artificial viscosity was used to separate dust and glass material, which differ highly in the "stiffness" of their equations of state. 

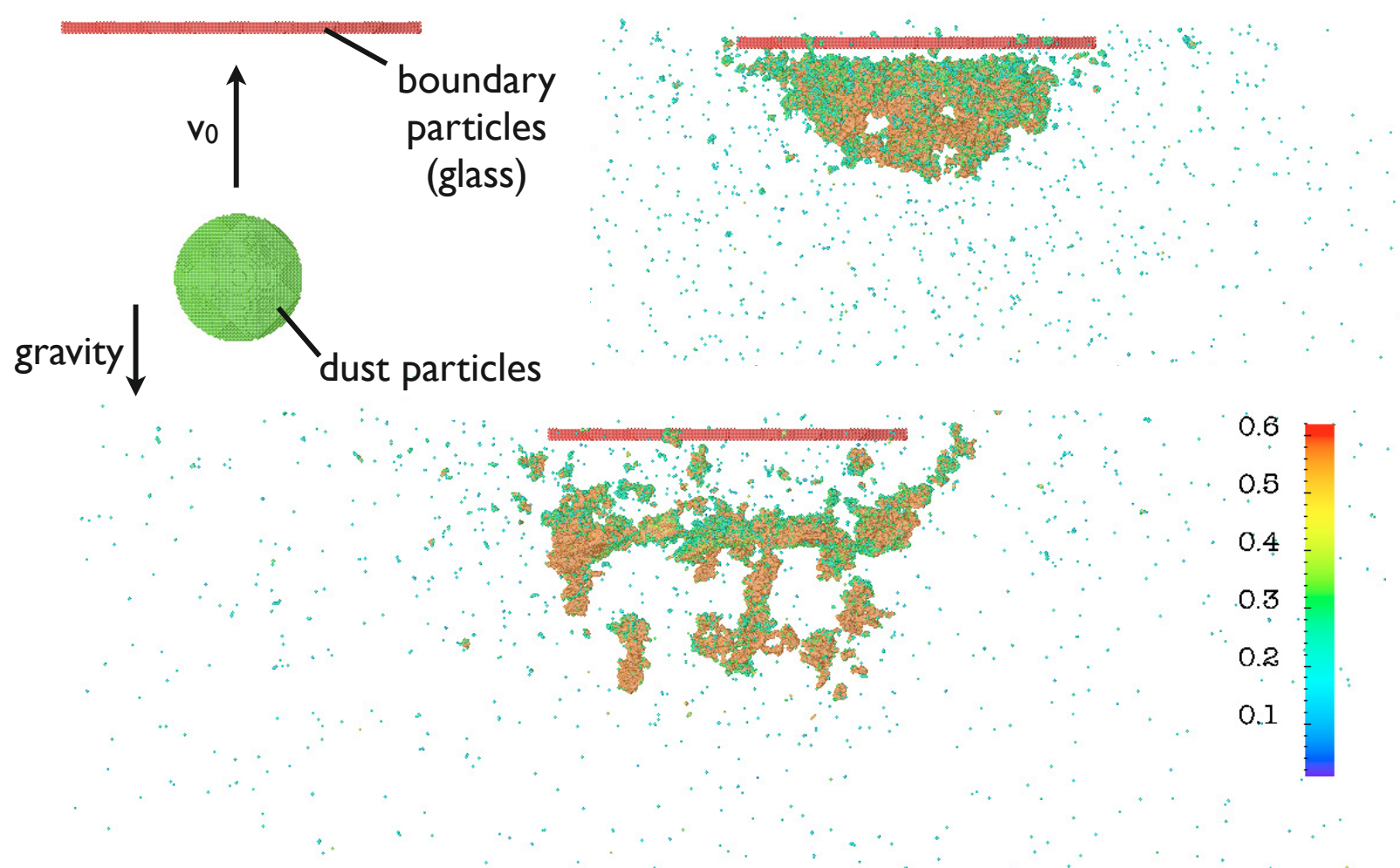

Fig. 20. Fragmentation sequence for $t=0 \mathrm{~ms}$ (top left), $t=0.4 \mathrm{~ms}$ (top right), and $t=0.8 \mathrm{~ms}$ (bottom). The colour code indicates the filling factor. A projectile consisting of dust particles (Sirono EOS, diameter $0.57 \mathrm{~mm}$ ) hits a solid surface simulated by boundary glass particles (Murnaghan EOS, diameter $1.6 \mathrm{~mm}$, thickness $0.04 \mathrm{~mm}$ ) with an impact velocity of $v_{0}=8.4 \mathrm{~m} \mathrm{~s}^{-1}$. A bulk modulus of $K_{0}=4.5 \mathrm{kPa}$ was used for this simulation. While many small pieces, often single SPH particles, are chipped off, most of the aggregate is compressed to its maximum filling factor $\phi_{\max }=0.58$ and fractures. The bottom layer of the impacting sphere is hardly compressed at all. This uncompressed layer remains visible in the fragments. (An animation of this figure is available in the online journal.)

We investigated the crucial differences between 2D and $3 \mathrm{D}$ compaction calibration setup and proved the validity of the correction factor for the intrusion depth (Eq. (26)) that was already used without confirmation in Güttler et al. (2009). The major difference from the experiments and simulations in Güttler et al. (2009) was that in that study compression reached too deep into the dust sample beneath the glass sphere and caused an excess of total compressed volume relative to the experiments. Both problems were resolved in this work by using the 3D setup.

Using a series of 2D simulations of the compaction calibration setup, we predicted a new, more accurate, value for the mean pressure $p_{\mathrm{m}}$ of the dynamic compressive strength relation. A 3D simulation with this value $p_{\mathrm{m}}=0.26 \mathrm{kPa}$ was carried out. The results were compared with the experimental reference using the same benchmark features as in Güttler et al. (2009). We found good agreement.

In Güttler et al. (2009), it was demonstrated that our code is in principle capable of simulating the bouncing of dust aggregates. With the bouncing calibration setup, we have investigated the ability of the SPH code to quantitatively simulate the elastic properties of the dust and the energy dissipation by compression. We found that the bulk modulus as well as the compressive strength relation have a significant influence. For lower values of $p_{\mathrm{m}}$ (low compressive strength) and higher values of the bulk modulus, more energy is dissipated. Using $p_{\mathrm{m}}=0.26 \mathrm{kPa}$ from the compaction calibration setup, we deduced that the uncompressed dust samples $(\phi \approx 0.15)$ have a bulk modulus $K_{0} \approx 5 \mathrm{kPa}$. With this result, we were able to decide between two differing experimental values in favour of the indirect determination by Weidling et al. (2009).

An important application of this code will be preplanetesimal collisions. We therefore also tested the code ability to simulate the fragmentation of dust aggregates quantitatively correctly. For this, we used a fragmentation calibration setup and identified the bulk modulus to be the quantity with the strongest influence on the fragment distribution. Using $p_{\mathrm{m}}=0.26 \mathrm{kPa}$ again from the compaction calibration setup, we found closest agreement with the empirical reference for the bulk modulus $K_{0}=4.5 \mathrm{kPa}$. Remarkably this is consistent with the findings of the bouncing calibration setup, which represents a test with a totally different behaviour. The problem of almost no fragmentation for $K_{0}=300 \mathrm{kPa}$ described in Güttler et al. (2009) was hereby traced back to an incorrect assumption about the bulk modulus.

\section{Conclusion}

Schäfer et al. (2007) demonstrated that the outcome of preplanetesimal collisions crucially depends on their material properties. If collisional growth is to be investigated by computer simulations, they suggested an implementation of experimentally measured material parameters and thorough calibration and comparison with laboratory experiments.

The SPH code presented in this work complies with their requirements. Based on the experimental preparatory work by Güttler et al. (2009), this code has been successfully tested with 
three kinds of calibration setups for the correct simulation of compaction, bouncing, and fragmentation.

We conclude that we have developed a tool that features a sufficient accuracy for the investigation of the outcome of preplanetesimal collisions in parameter ranges that are not accessible to laboratory experiments. This is based on the assumption that the macroscopic properties calibrated for in this work do not change with increasing size. However, due to the thermodynamically simple porosity model used in the SPH code the area of application is restricted to a certain range of collision velocities. Collisions in which shock propagation cannot be neglected are outside the physically meaningful limit of this model. Changes in the mechanical properties with temperature and other thermodynamical effects such as sintering and melting cannot be simulated. An extension of the porosity model and its implementation and calibration are necessary to broaden the parameter range (e.g., supersonic impacts) and to consider a realistic environment for pre-planetesimal collisions in the protoplanetary disc.

Nevertheless, within its area of application our code will be used to produce a "catalogue of collisions". The influence of object sizes, porosity, relative velocity, rotation, and impact parameter on the fragment distribution of pre-planetesimal collisions will be investigated in future work.

Acknowledgements. The authors want to thank Serena E. Arena for many fruitful discussions and helpful comments about the manuscript. The SPH simulations were performed on the university and bwGriD clusters of the computing centre (ZDV) of the University of Tübingen. We thank M.-B. Kallenrode and the University of Osnabrück for providing access to the XRT setup. We also thank the anonymous referee for clarifying comments and suggestions. This project was funded by the Deutsche Forschungsgemeinschaft within the Forschergruppe 759 "The Formation of Planets: The Critical First Growth Phase" under grants B1 298/7-1, B1 298/8-1, and K1 650/8-1.

\section{References}

Blum, J., \& Münch, M. 1993, Icarus, 106, 151

Benz, W., \& Asphaug, E. 1994, Icarus, 107, 98

Benz, W., \& Asphaug, E. 1995, Comput. Phys. Commun., 87, 253

Blum, J., \& Schräpler, R. 2004, Phys. Rev. Lett., 93, 115503

Blum, J., \& Wurm, G. 2000, Icarus, 143, 138

Blum, J., \& Wurm, G. 2008, ARA\&A, 46, 21

Blum, J., Wurm, G., Kempf, S., et al. 2000, Phys. Rev. Lett., 85, 2426

Blum, J., Schräpler, R., Davidsson, B. J. R., \& Trigo-Rodríguez, J. M. 2006, ApJ, 652, 1768

Brauer, F., Dullemond, C. P., \& Henning, T. 2008, A\&A, 480, 859

Davis, D. R., \& Ryan, E. V. 1990, Icarus, 83, 156

Dominik, C., \& Tielens, A. G. G. M. 1997, ApJ, 480, 647

Dullemond, C. P., \& Dominik, C. 2005, A\&A, 434, 971

Geretshauser, R. 2006, Master's thesis, Eberhard-Karls-Universität Tübingen

Gingold, R. A., \& Monaghan, J. J. 1977, MNRAS, 181, 375
Grady, D. E., \& Kipp, M. E. 1980, Int. J. Rock Mech. Min. Sci. Geomech. Abstr., 17,147

Güttler, C., Krause, M., Geretshauser, R. J., Speith, R., \& Blum, J. 2009, ApJ, 701,130

Güttler, C., Blum, J., Zsom, A., Ormel, C. W., \& Dullemond, C. P. 2010, A\&A, 513, A56

Heim, L.-O., Blum, J., Preuss, M., \& Butt, H.-J. 1999, Phys. Rev. Lett., 83, 3328 Heißelmann, D., Fraser, H., \& Blum, J. 2007, in Proceedings of the 58th International Astronautical Congress 2007, IAC-07-A2.1.02

Jutzi, M. 2008, Ph.D. thesis, Universität Bern

Jutzi, M., Benz, W., \& Michel, P. 2008, Icarus, 198, 242

Jutzi, M., Michel, P., Hiraoka, K., Nakamura, A. M., \& Benz, W. 2009, Icarus, 201,802

Krause, M., \& Blum, J. 2004, Phys. Rev. Lett., 93, 021103

Libersky, L. D., \& Petschek, A. G. 1990, in Advances in the Free-Lagrange Method, ed. H. E. Trease, M. J. Fritts, \& W. P. Crowley (Springer Verlag), 248

Libersky, L. D., Petschek, A. G., Carney, T. C., Hipp, J. R., \& Allahdadi, F. A. 1993, J. Chem. Phys., 109, 67

Libersky, L. D., Randles, P. W., Carney, T. C., \& Dickinson, D. L. 1997, Int. J. Impact Eng., 20, 525

Lucy, L. B. 1977, ApJ, 82, 1013

Mathis, J. S., Rumpl, W., \& Nordsieck, K. H. 1977, ApJ, 217, 425

Melosh, H. J. 1989, Impact cratering: A geologic process, Oxford monographs on geology and geophysics, ed. H. J. Melosh (Oxford University Press), 11

Monaghan, J. J. 2005, Rep. Prog. Phys., 68, 1703

Monaghan, J. J., \& Gingold, R. A. 1983, J. Chem. Phys., 52, 374

Monaghan, J. J., \& Lattanzio, J. C. 1985, A\&A, 149, 135

Omang, M., Børve, S., \& Trulsen, J. 2006, J. Comput. Phys., 213, 391

Ormel, C. W., Spaans, M., \& Tielens, A. G. G. M. 2007, A\&A, 461, 215

Ormel, C. W., Paszun, D., Dominik, C., \& Tielens, A. G. G. M. 2009, A\&A, 502, 845

Paszun, D., \& Dominik, C. 2006, Icarus, 182, 274

Paszun, D., \& Dominik, C. 2008, A\&A, 484, 859

Paszun, D., \& Dominik, C. 2009, A\&A, 507, 1023

Randles, P. W., \& Libersky, L. D. 1996, Comp. Methods Appl. Mech. Engrg, 139,375

Schäfer, C., Speith, R., \& Kley, W. 2007, A\&A, 470, 733

Sirono, S.-I. 2004, Icarus, 167, 431

Teiser, J., \& Wurm, G. 2009, MNRAS, 393, 1584

Wada, K., Tanaka, H., Suyama, T., Kimura, H., \& Yamamoto, T. 2007, ApJ, 661, 320

Wada, K., Tanaka, H., Suyama, T., Kimura, H., \& Yamamoto, T. 2008, ApJ, 677, 1296

Wada, K., Tanaka, H., Suyama, T., Kimura, H., \& Yamamoto, T. 2009, ApJ, in press

Weidenschilling, S. J. 1977, MNRAS, 180, 57

Weidenschilling, S. J., \& Cuzzi, J. N. 1993, in Protostars and Planets III, ed. E. H. Levy, \& J. I. Lunine, 1031

Weidenschilling, S. J., Spaute, D., Davis, D. R., Marzari, F., \& Ohtsuki, K. 1997, Icarus, 128,429

Weidling, R., Güttler, C., Blum, J., \& Brauer, F. 2009, ApJ, 696, 2036

Wurm, G., Paraskov, G., \& Krauss, O. 2005, Icarus, 178, 253

Zsom, A., \& Dullemond, C. P. 2008, A\&A, 489, 931

Zsom, A., Ormel, C. W., Güttler, C., Blum, J., \& Dullemond, C. P. 2010, A\&A, 513, A57 